نمذجة الاقتصاد الخفي وعجز الموازنة العامة في مصر

د. حسام الدين محمد عبد القادر

\author{
مدرس الاقتصاد \\ كلية التجارة - جامعة عين شمس الافهي
}

1 ا مقدمة

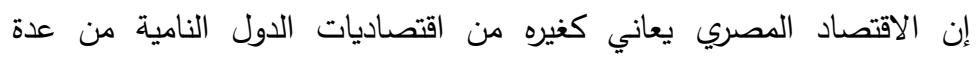

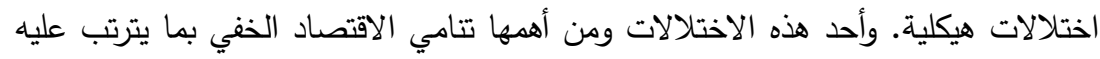

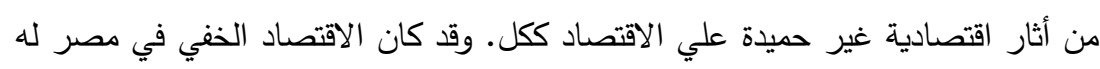

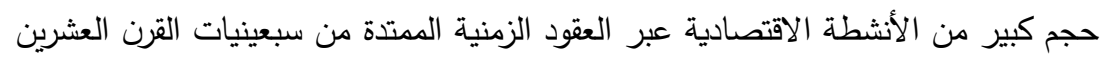

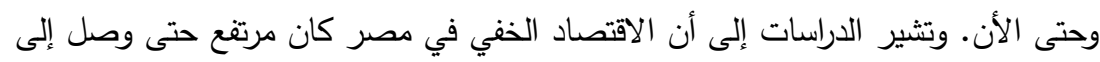

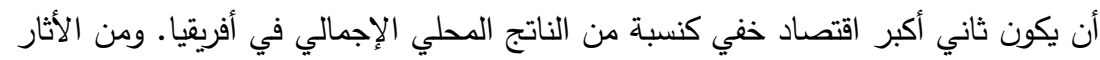

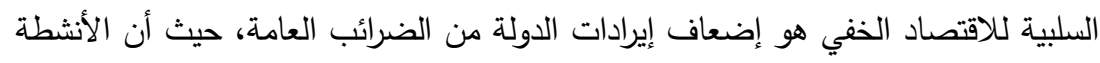

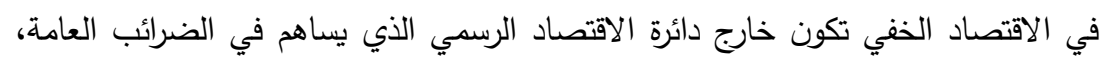
كما أن الأنشطة في الاقتصاد الخفي تستفيد من بعض أوجه الإنفاق العام في الدولة.

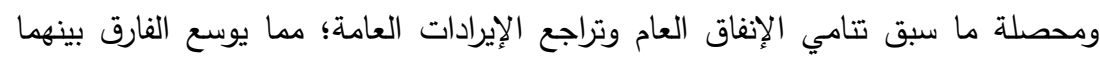

ويفاقم من العجز في الموازنة العامة للدولة.

ورغم أهمية قضية الاقتصاد الخفي في الاقتصاد المصري إلا أن الدراسات التي لإني

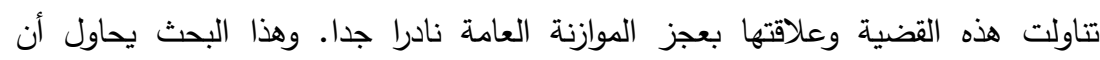
يستكثف العلاقة ما بين الاقتصاد الخفي وعجز الموازنة العامة للدولة في مصر خلاد الفتله الفترة

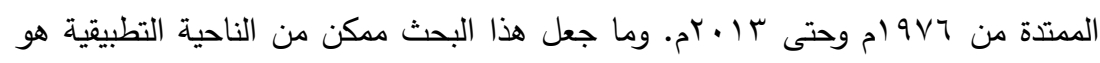

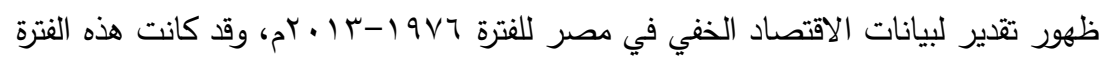
هي الحدود الزمنية للدراسة.

وفي هذه الدراسة يتم التركيز على الاقتصاد الحفي في مصر ومحاولة اختبار

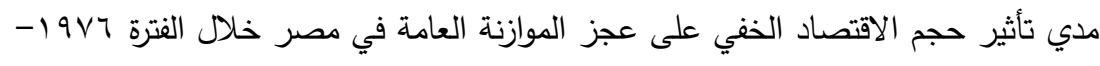


r ا. بم. ويمكن استعراض فرض الدراسة الأساسي التي تحاول اختباره هو إن تنزيد حجم

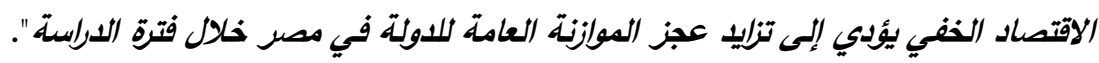

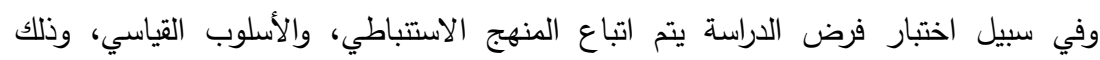

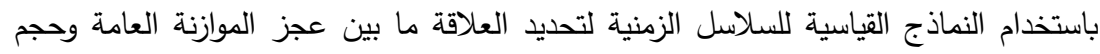

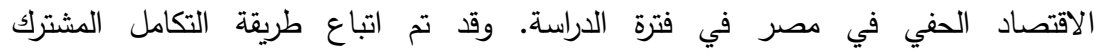
Cointegration

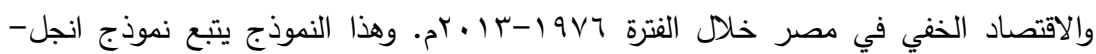
جرانجر ذو الخطوتين Engle-Granger Two-Step Model.

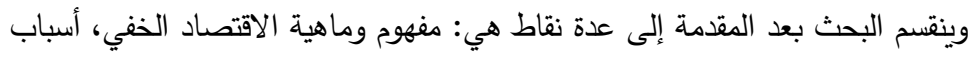

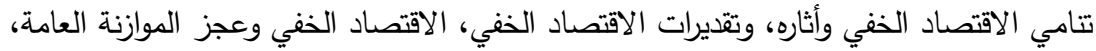

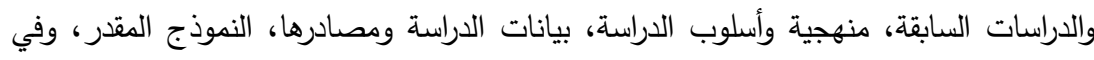
النهاية نتائج وتوصيات الدراسة.

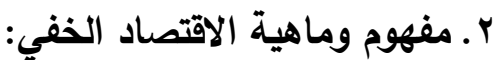

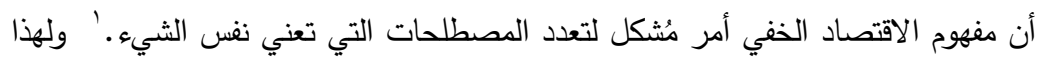

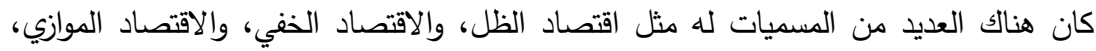

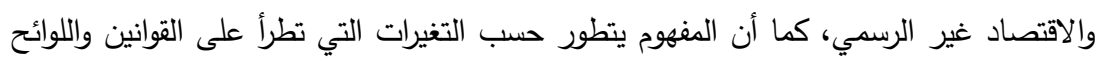

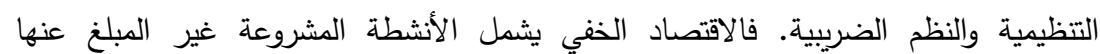
ضريبيا، وكنلك الأنشطة غير المشروعة كما يتضح من الجدول (1).

'لهناك العديد من المصطلحات المستخذمة للتدليل على نفس المصطلح وهي الاقتصاد الخفي

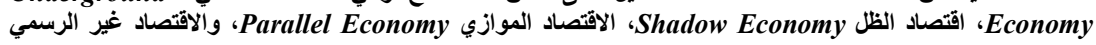


جدول (1)

\begin{tabular}{|c|c|c|}
\hline المعاملات غير النقدية & المعاملات النقدية & نوع النشاط \\
\hline 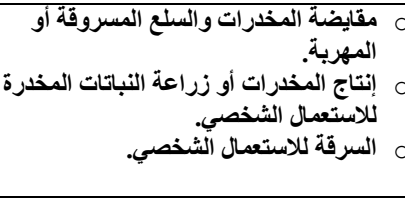 & c الاتجار في الاتجار في المخلع المسروقة. & الأنشطة غير \\
\hline التجنب الضريبي & التهرب الضريبي & نوع النشاط \\
\hline 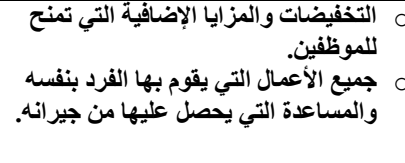 & 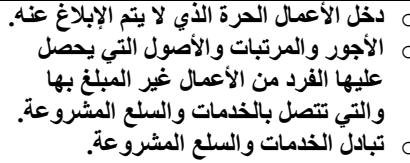 & الأثثطة المشروعة \\
\hline
\end{tabular}

Source: Schneider, Friedrich and Enste, Dominik (2002) Hiding in the Shadows: The
Growth of the Underground Economy, IMF Economic Issues, No. 30, March, p.2

ويمكن تعريف الاقتصاد الخفي بانه "جميع الأنشطة الاقتصادية التي تخضع

للضريبة بشكل عام إذا ما أُبلغت بها السلطات الضريبية". كما يعرف الاقتصاد الخفي بأنه الأنه

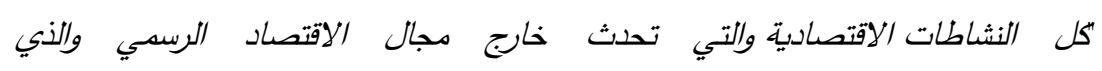

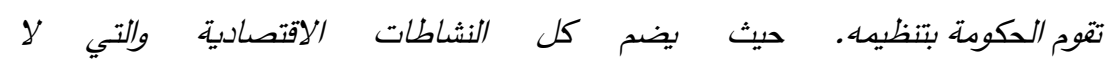

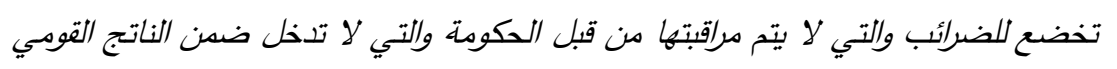

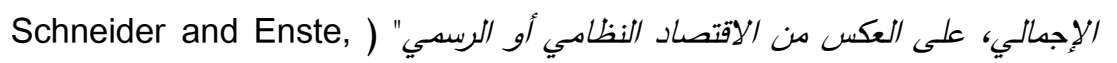

.(2002, p.2

وقد يتبادر إلى الذهن أن الاقتصاد الخفي هو خاصية تميز الاقتصاديات والدول

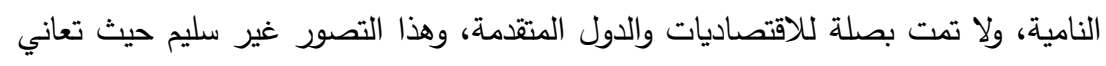
كافة دول العالم من وجود الاقتصاد الخفي بها، رغم أن حجم هذا القطاع يتفاوتاً تفوت كبيراً

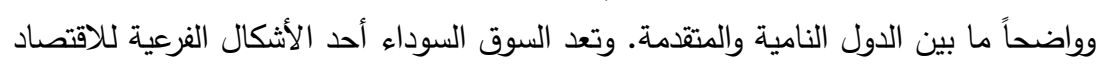

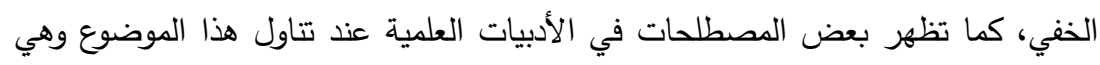

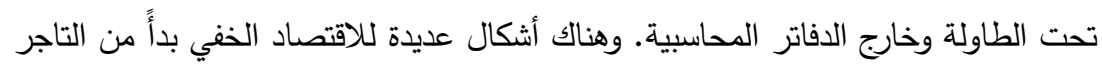


الذي يتهرب أو يتجنب الضريبة، وسائق التاكسي الذي يقوم بالقيادة بدون رخصة، والموظف المرتشي، وحتى المهربين، وتجار العملة، وتجار المخدرات والدعارة. ويظهر في الجدول

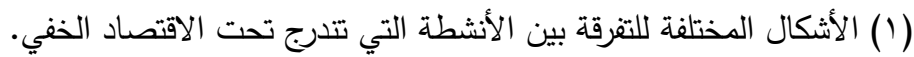

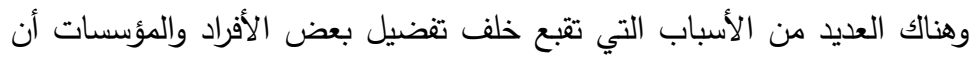

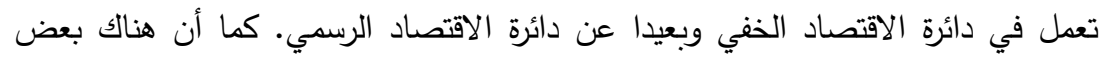
الصعوبات التي تواجه المشروعات التي تعمل في ظل الاقتصاد الخفي، وفي أحد الدراسات

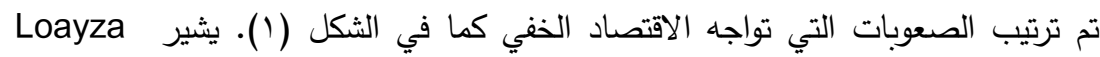

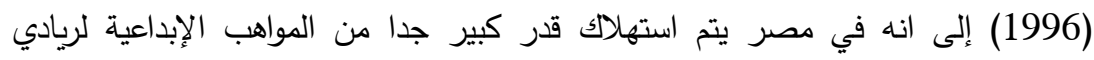

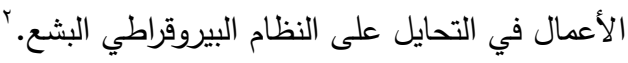

\section{(1) (1)}

الأهمية النسبية (\%) للصعوبات التي تواجه المشروعات في الاقتصاد الخفي

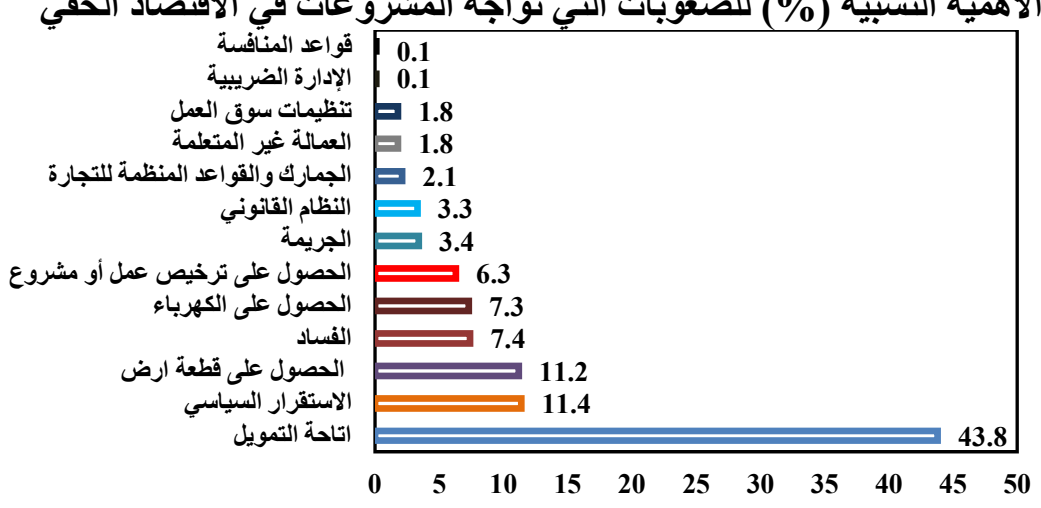

Source: Porta, Rafael La and Shleifer, Andrei (2014) Informality and Development, Journal of Economic Perspectives, Vol. 28, No., P.1 • 9-126.

\section{r. أسباب تنامي الاقتصاد الخفي وأثاره الاقتصادية}

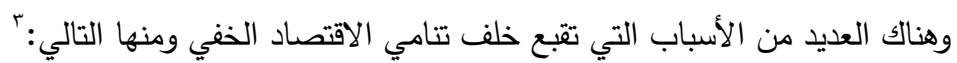

2 "In Egypt, much of the country's entrepreneurial talent is consumed in circumventing the country's nightmare bureaucratic regulatory system"

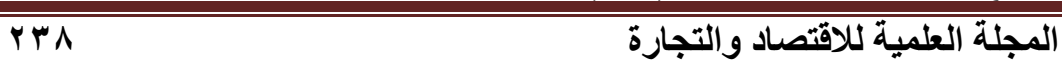


ا. إن تنامي الأعباء الضريبية ومدفوعات الضمان الاجتماعي، وقيود سوق العمل وتدني الإني

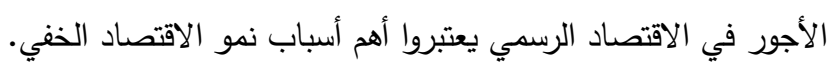

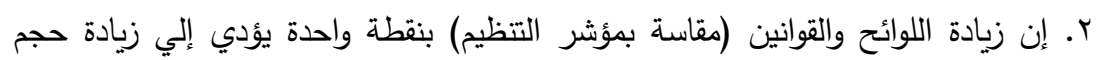

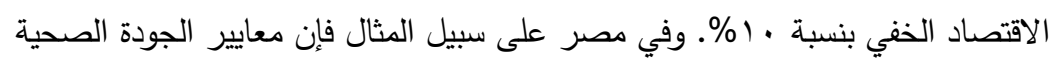

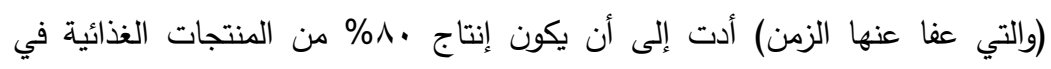
الاقتصاد الخفي، بأحجام مشروعات صغير ومنخفضة الإنتاجية.

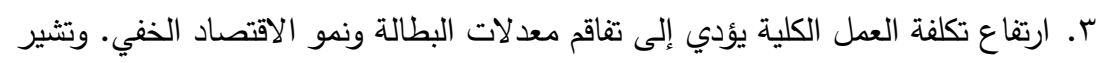

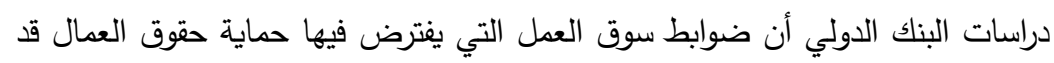

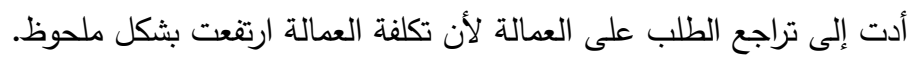

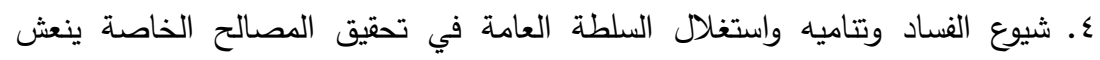

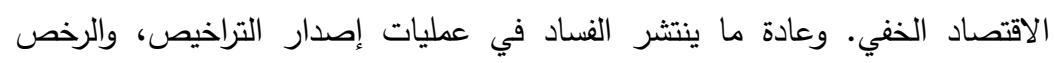

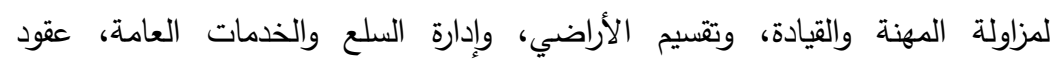
الاستثمار ، وتحصيل وربط الضرائب، والتعينات والترقيات.

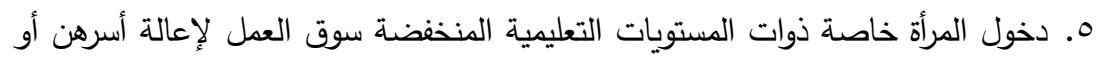

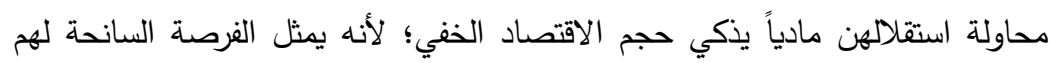

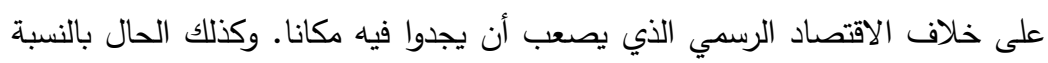

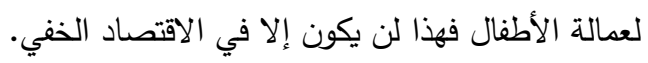

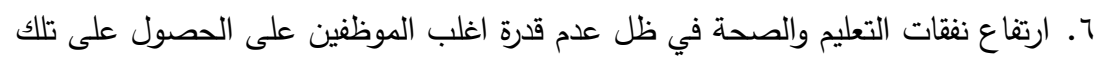

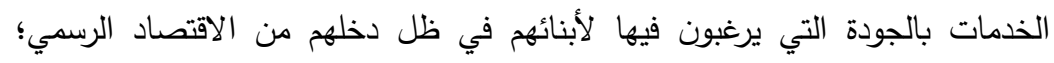

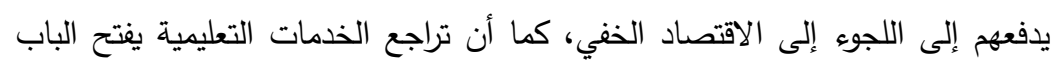
على مصراعيه لإذكاء الاقتصاد الخفي في سوق الدروس الخصاد الخصوصية. كما أن تنامي الاقتصاد الخفي ينشأ عنه العليد من الأثار الأقصادية ومن أهمها ما يلي؛ 
ا. تنامي الاقتصاد الخفي يضعف مصداقية الإحصاءات الرسمية عن الناتج والاستهلاك، والتوظف والبطالة، ومن ثم تكون السياسات الاقتصادية الكلية المطبقة غير فعالة، بل الل الإل وقد تكون غير ذي جدوى (Schneider and Enste, 2002). r. نمو الاقتصاد الخفي يؤثر سلباً على معدلات النمو الاقتصادي، ومسيرة التنمية في الدولة وخاصة الاقتصاد الرسمي. r. إن التجنب والتهرب الضريبي الحادث في الاقتصاد الخفي يهمش الحصيلة الضريبية،

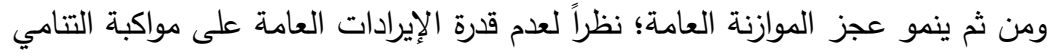

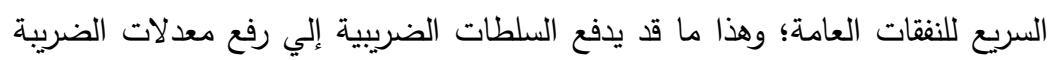

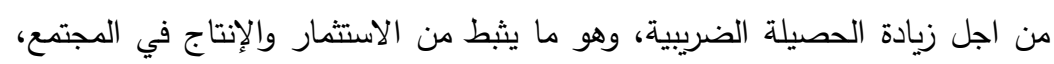
كما انه يغري الكثيرين بالعمل في ظل الاقتصاد الخفي لتجنب الضرائب أو التهرب منها

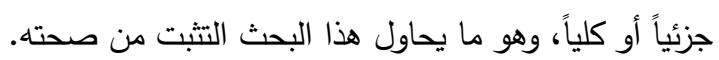

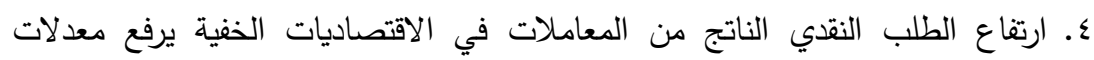
التضخم؛ مما يخفض من رفاهية الأفراد في المجتمع.

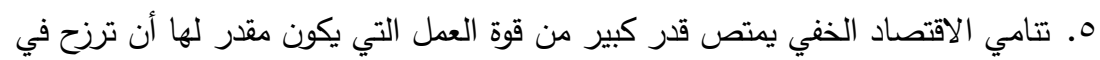
معترك البطالة، في ظل عدم قدرة القطاع الرسمي (الحكومي والخاص) على توفير القدر الكافي من فرص العمل (Schneider and Enste, 2002)؛ إلا أن هذه العمالة تكون غير مستقرة، وإنتاجيتها منخفضة، وفرصها في التوسع المستقبلي تكاد تكون منعدمة.

\section{ـ ـققديرات حجم الاقتصاد الخفي في الاقتصاد المصري}

إن تحديد حجم الاقتصاد الخفي يعد الخطوة الأولي لمعرفة خطورة هذه الظاهرة

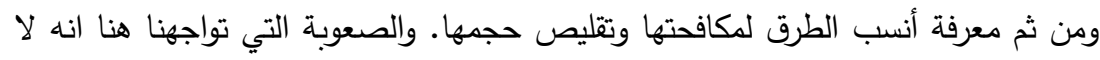
يوجد إحصاءات رسمية دقيقة عن هذه الظاهرة، ومن ثم يتم اللجوء إلى تقدير حجم الاقتصاد الخفي، وتتعدد طرق تقدير وقياس حجم الاقتصاد الخفي، كما في الجدول (ץ). 


\section{جدول (r) ( )}

\begin{tabular}{|c|c|}
\hline المناهج غير المباــرة & المناهج المبإرة \\
\hline 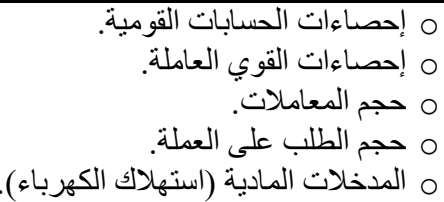 & م م المقيق الحسابات الضرينة. \\
\hline
\end{tabular}

Source: Schneider, Friedrich and Enste, Dominik (2002) Hiding in the Shadows: The Growth of the Underground Economy, IMF Economic Issues, No. 30, March, p.12.

\section{أ. أ. حجم الاقتصاد الخفي من الناتج المحلي الإجمالي}

في دراسة (2000) Schneider \& Enste تم تقدير حجم الاقتصاد الخفي في

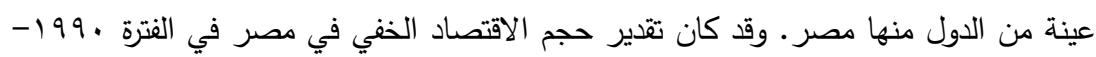

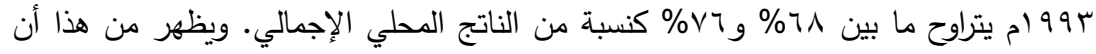

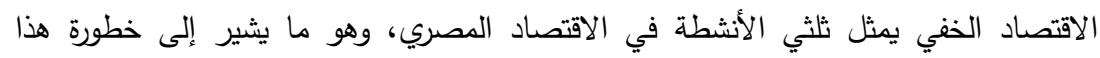

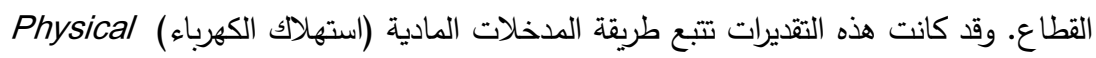
Inputs (Electricity)

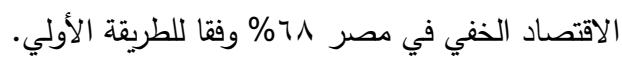
هناك العديد من التقديرات لحجم الاقتصاد الخفي في العديد من الدول نظرا لعدم توافر

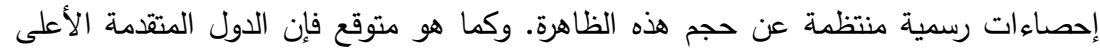

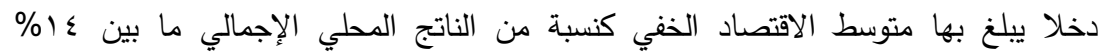

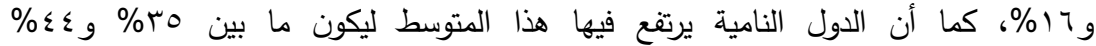
Schneider and Enste, 2002, p.3)

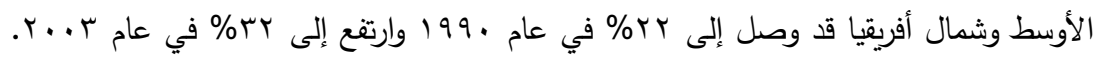

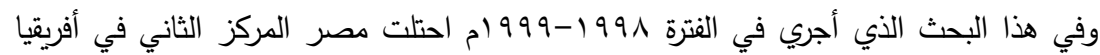
بعد نيجيريا مباشرة في حجم الاقتصاد الخفي كنسبة من الناتج المحلي الإجمالي؛ حيث كانت النافي

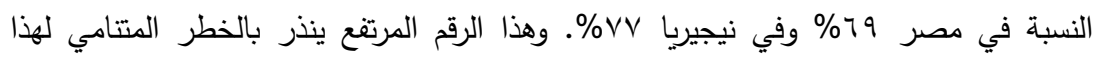
الاقتصاد الخفي في كلا الدولتين(Schneider and Enste, 2002, p.4) . 
استخدم Schneider (2005) طريقة المؤشرات المتعددة والمسببات المتعددة

الديناميكية (DYMIMIC) تقدير حجم الاقتصاد الخفي في عينة من الدول منها مصر • ووفقا لتقديرات البحث فان الاقتصاد

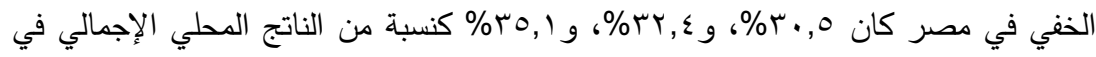

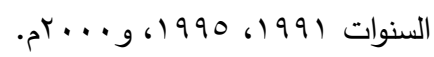

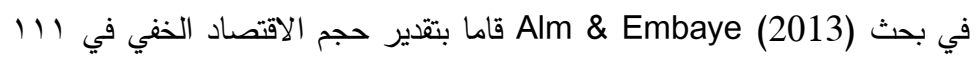

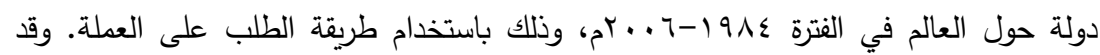
كانت مصر من دول العينة حيث كان حجم الاقتصاد الخفي فيها كنسبة من الناتج المحلي

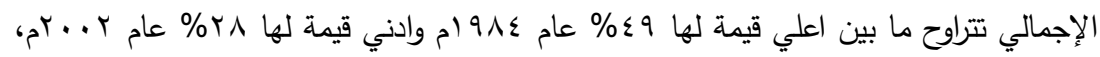

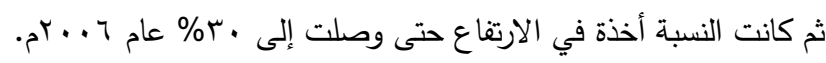

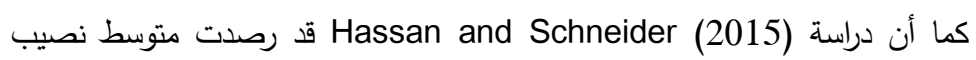

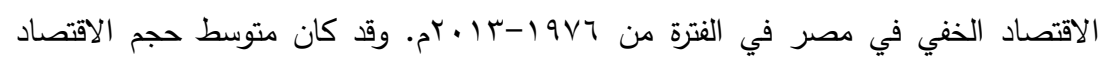

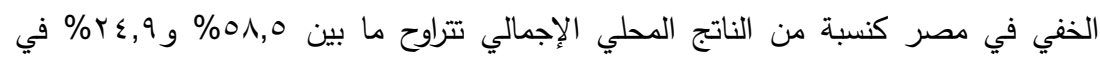

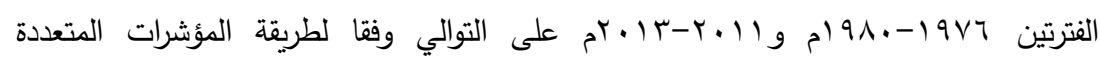
والمسببات المتعددة MIMIC. أما في حالة استخدام طريقة الطلب على العملة CDA فان

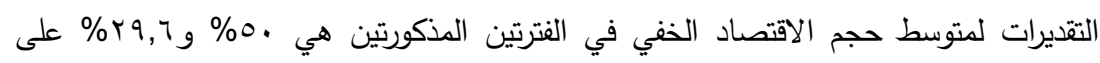
التوالي؛ وهو ما يشير إلى الأهمية النسبية للاقتصاد الخفي في مصر .

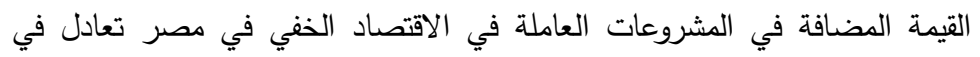
المتوسط • r\% من القيمة المضافة في الشركات المماثلة والعاملة في الاقتصاد الرسمي. وفي حالة المقارنة مع الثركات صغيرة الحجم نجد أن القيمة المضافة بالنسبة للمشروعات في الاقتصاد الخفي هي VIV) (La Porta and Shleifer, 2014, pp. 113-114). ب. حجم الاقتصاد الخفي في قوة العمل كما يتضح من الجدول (ب) هنالك فارق كبير ما بين العمالة في الاقتصاد الخفي والاقتصاد الرسمي، حيث ينخفض متوسط عدد العمالة في الاقتصاد الخفي للمشروع بنسبة كبيرة جدا مقارنة بالاقتصاد الرسمي. 


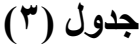

خصائص العمل في الاقتصاد الخفي مقارنة بالاقتصاد الرسمي

\begin{tabular}{|c|c|c|}
\hline الاقتصاد الخفي & الاقتصاد الرسمي & أوجه المقارنة \\
\hline ؛ عمال. & Tץ T ו عامل. & متوسط عدد العمالة في المشروع الواحد. \\
\hline في الاقتصاد الرسمي. المضافة & متوسطة أو عالية. & القيمة المضافة للعامل. \\
\hline$\% \vee$ & $\% \vee 7$ & المديرين الحاصلين على مؤهل جامعي. \\
\hline$\% 0^{\circ}$ & $\% 1$. & عدل نمو التوظف سنويا. \\
\hline
\end{tabular}

Source: Porta, Rafael La and Shleifer, Andrei (2014) Informality and Development, Journal of Economic Perspectives, Vol. 28, No., P.1·9-126.

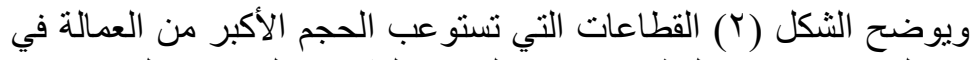

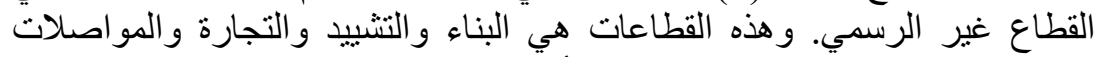
و التصنيع ثم الخدمات علي حسب نرتيب أهنيتهم في استيعاب العمالة.

(r) (r)

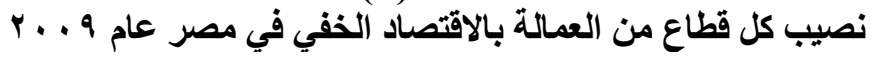

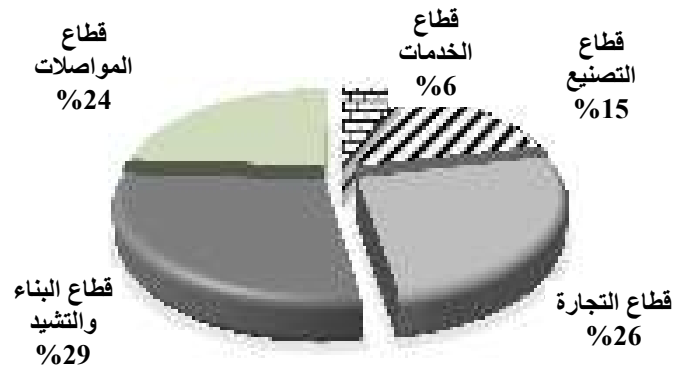

Sources: ILO and WIEGO Databases

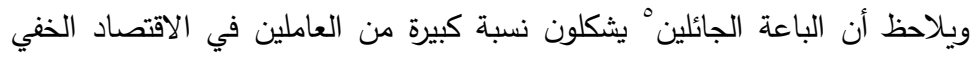

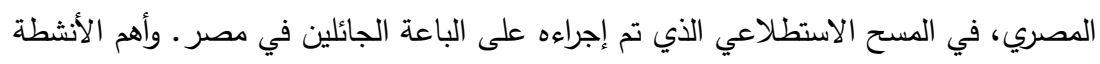

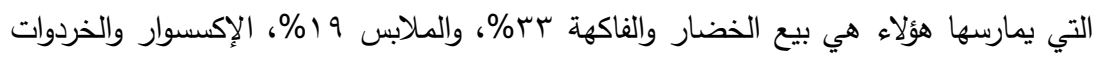

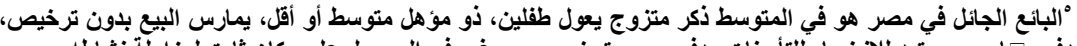

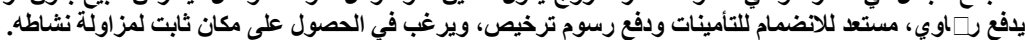

$r \varepsilon r$

المجلة العلمية للاقتصاد و التجارة 
9\%، والمأكولات والمشروبات ^^\%، والأحذية والمصنوعات الجلدية ^\%، والبقالة والسجائر

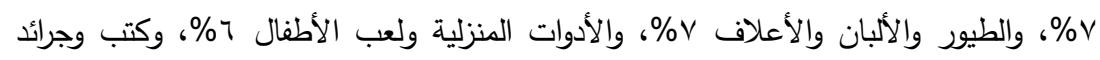

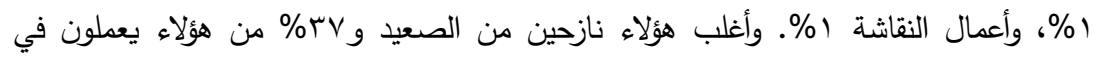

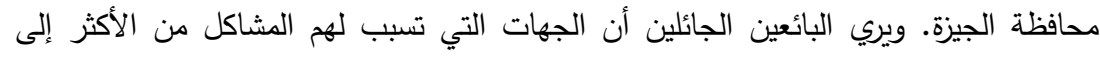

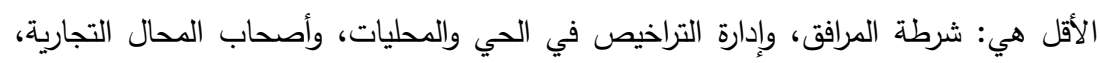

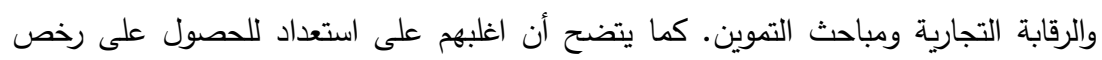

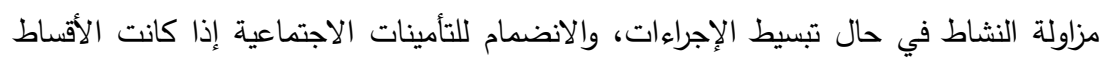

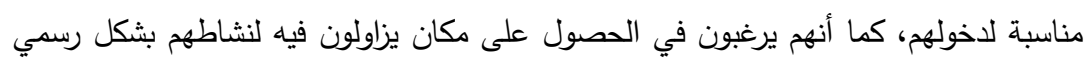

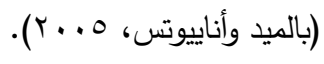

\section{هـ الاقتصاد الخفي وعجز الموازنة العامة}

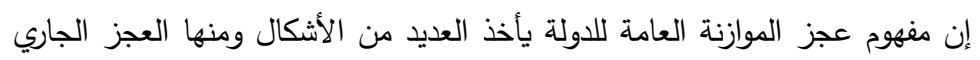

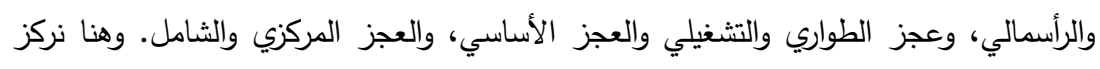

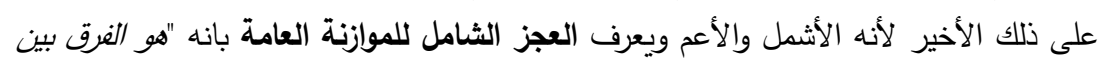

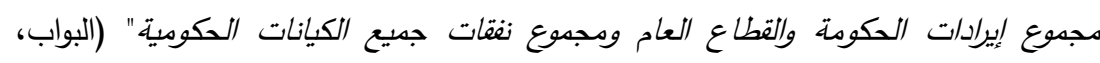
'. ( r... إن غياب مرونة الموازنة العامة بالنسبة للتعديلات في الإنفاق العام ورفع معدلات

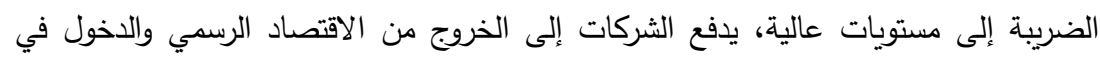

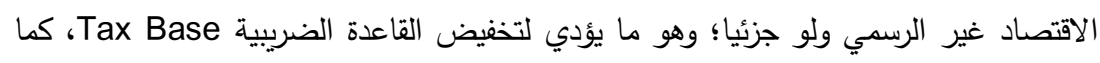

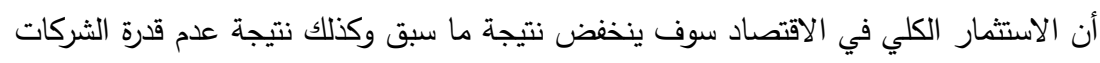

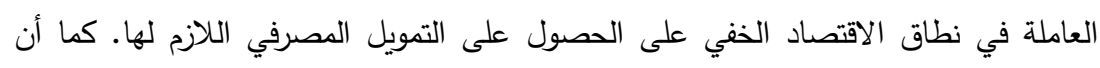

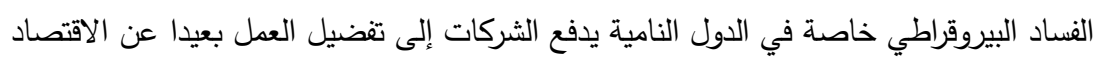

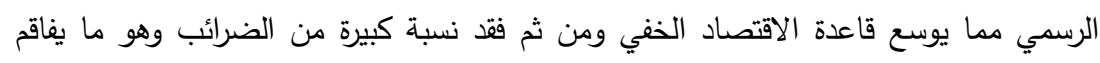
عجز الموازنة العامة (Norris \& Feldstein, 2003).

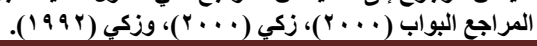


وبالنظر إلى أبسط شكل للموازنة العامة للدولة بهدف تحديد حجم عجز الموازنة العامة BDt فانه سوف يمثل الفرق ما بين جانب النفقات العامة والايرادات العامة. ويتكون جانب النفقات العامة من مجموع المشتريات الحكومية

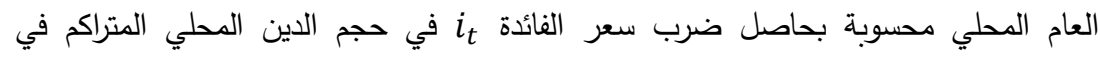
السنوات السابقة الدين الخارجي

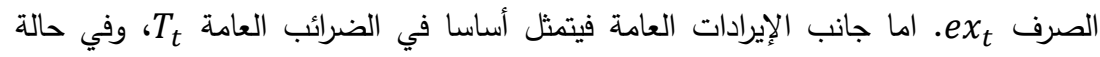
زيادة جانب النفقات عن الإيرادات سوف يكون هنالك عجز في الموازنة العامة للدول \&orris \& Feldstein, 2003)

$$
B D_{t}=G_{t}+S_{t}+i_{t} B_{t-1}+i_{F, t} e x_{t} B_{F, t-1}-T_{t}
$$

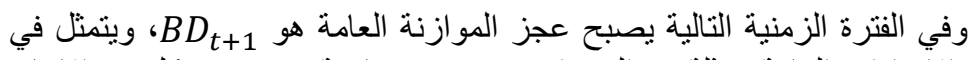
الفارق بين الإير ادات العامة ممثلة في الضرة الزئب

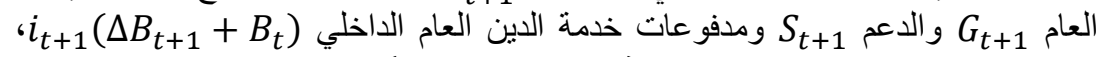
ومدفو عات خدمة الدين العام الخارجي (r)

$$
\begin{aligned}
B D_{t+1}=G_{t+1}+ & S_{t+1}+i_{t+1}\left(\Delta B_{t+1}+B_{t}\right) \\
& +i_{F, t+1} e x_{t+1}\left(\Delta B_{F, t+1}+B_{F, t}\right)-T_{t+1}
\end{aligned}
$$

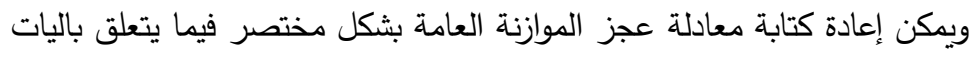

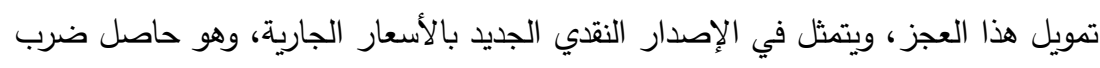

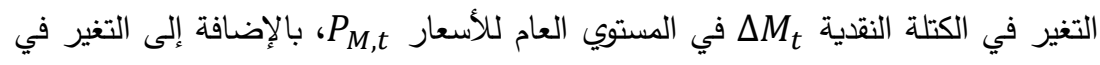
حجم الدين العام الداخلي الاسمي

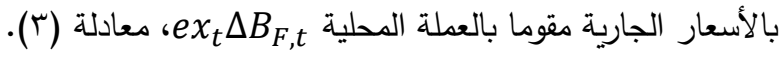

$$
B D_{t}=P_{M, t} \Delta M_{t}+P_{B, t} \Delta B_{t}+e x_{t} \Delta B_{F, t}
$$

ومن المعادلات السابقة يتضح أن حجم العجز في الموازنة العامة ينأثر بالتغيرات في إجمالي الإنفاق العام (الإنفاق العام والدعم) وهذه تتأثر بحجم الناتج الدحلي الإجمالي لإني الذي يعبر عن حجم النشاط الاقتصادي الكلي في الاقتصاد. كما يتأثر حجم الإنمان عجز الموازنة 
العامة بالتغير في الإيرادات العامة (الضرائب) وهذه الأخيرة تتأثر بتغيرات حجم الاقتصاد

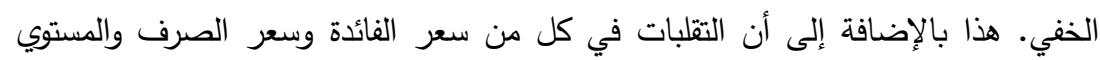
العام للأسعار. وهذه المتغيرات هي ما سوف يتم الاعتماد عليها في الجانب التطبيقي

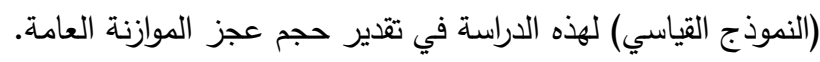

7. 1الاراسات السابقة

وفقا لغرض هذه الدراسة فيجب أن يتم الاسترشاد بالدراسات السابقة، ولكن عدد

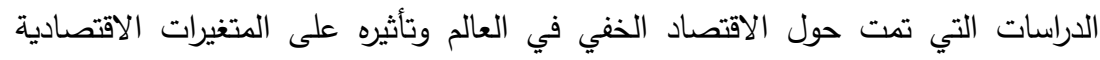

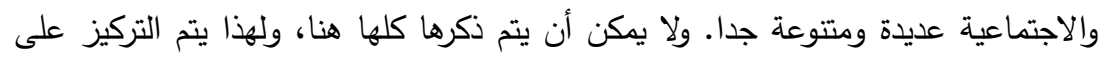
الدراسات السابقة ذات الصلة المباشرة فيما يتعلق بتأثير التغيرات في حجم الاقتصاد الخفي على التغيرات الحادثة في حجم عجز الموازنة العامة فقط.

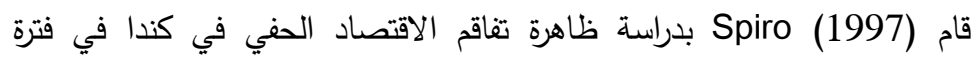
الثمانينيات والتسعينيات من القرن العشرين. وقد أشار إلي أن هناك علاقات تشابكية ما بين الأهين

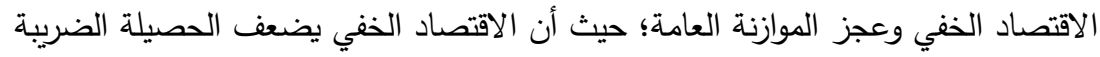

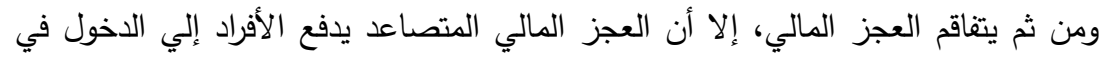

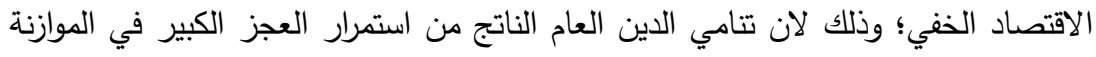

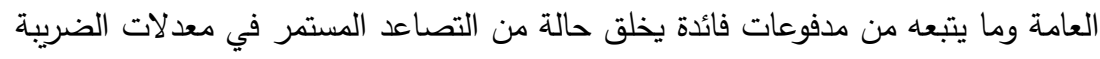

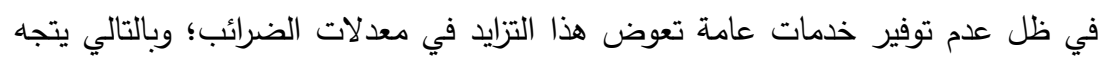
الأفراد إلي الولوج في الاقتصاد الخفي والابتعاد عن الاقتصاد الرسمي. قام (2003) Savaşan باستخدام طريقة المؤشرات المتعددة والمسببات المتعددة لتقدير حجم الاقتصاد الخفي في تركيا في الفترة • •9VIMIC

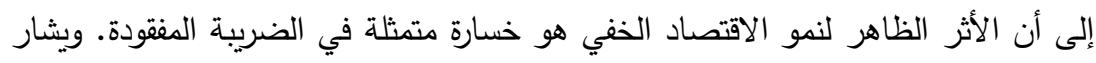
إلى هذا الفقد في الضرائب الناتج عن تنامي الاقتصاد الخفي بالفجوة الضريبة Tax Gap.

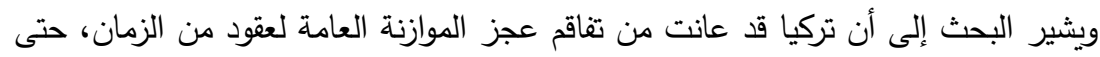

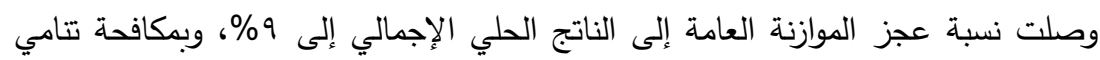
الاقتصاد الخفي في تركيا أمكن السيطرة على عجز الموازنة العامة بها. 
في دراسة (2011) Çiçek \& Elgin تم اختبار العلاقة ما بين السياسة المالية

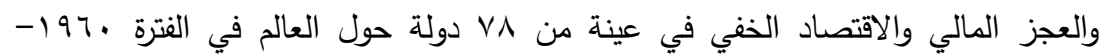

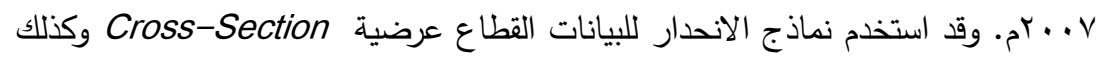

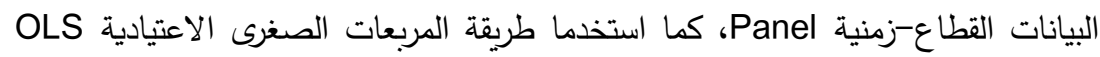

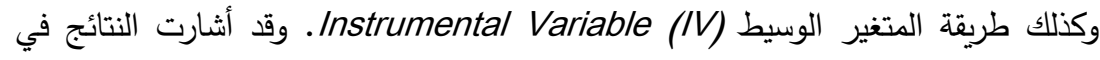

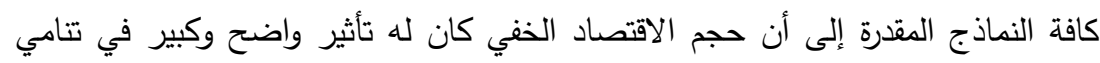
العجز المالي في دول العينة وقد كان تأثيره معنوي إحصائيا في كافة النماذج.

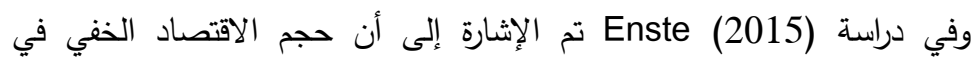

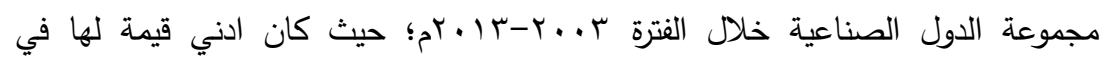

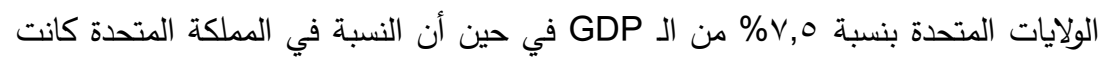

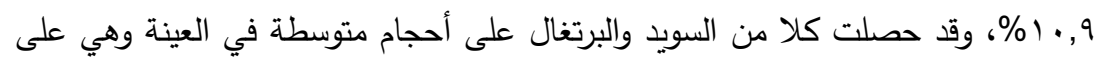

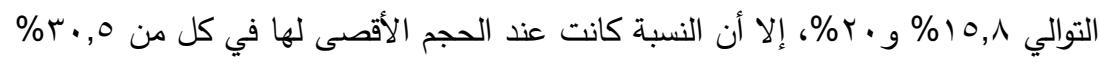

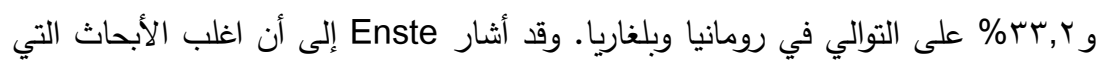

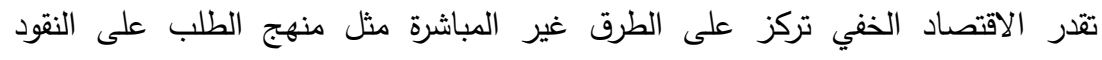
Monetary Demand Approach Multiple Indicators, Multiple Causes Approach (MIMIC) زيادة معدل الضريبة قد تدفع إلي زيادة حجم الاقتصاد الخفي، وهذا يدفع عجز الموازنة العامة إلى الارتفاع.

\section{V. منهجية وأسلوب الدراسة}

يقوم هذا البحث على اتباع المنهج الاستتباطي؛ حيث يحاول أن يختبر معطيات

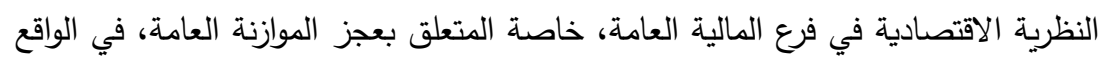
الاقتصادي المصري؛ وذلك بغرض معرفة مدي تأثير حجم الاقتصاد الخفي على استفحال

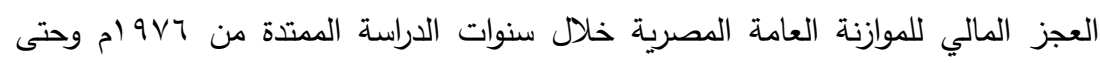

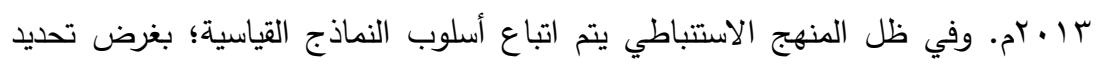

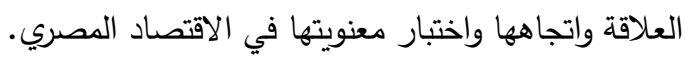


ووفقا للبيانات المتاحة وهي سلاسل زمنية سوف يكون نموذج الانحدار المستخدم

Autoregressive Distributed هنا لتقدير العلاقة يتبع نماذج الانحدار الذاتي المبطاء Lag Models (ADL)

$$
Y_{t}=\alpha+\sum_{j=1}^{J} \beta_{j} Y_{t-j}+\sum_{i=1}^{N} \sum_{k=0}^{K} \gamma_{i, k} \boldsymbol{X}_{i, t-k}+\epsilon_{t}
$$

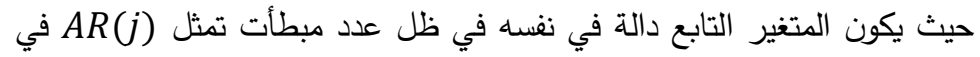

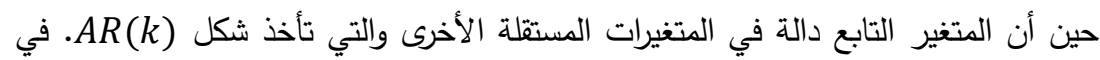

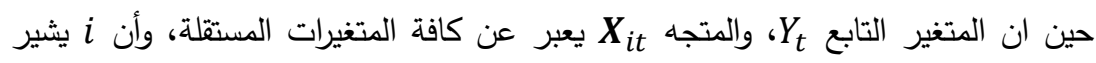

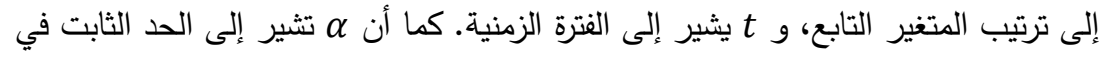

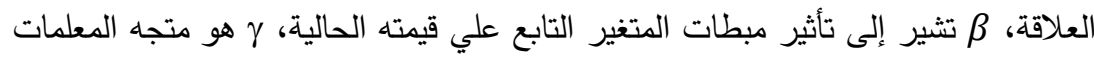

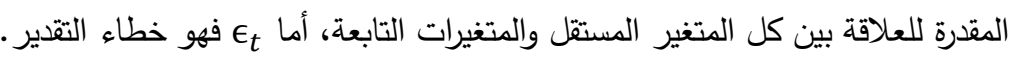

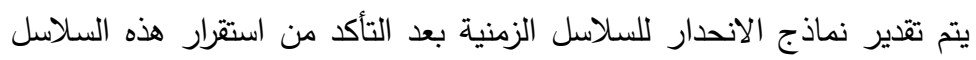
الزمنية Stationarity. ويكون ذلك باستخدام اختبارات جذور الوحدة Unit Root Tests. ولعل أحد أشهر هذه الاختبارات هو اختبار ديكي-فوللر (Dickey \& Fuller, 1979)،

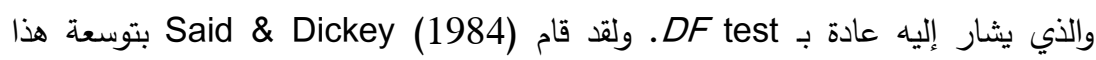
Autoregressive Unit Root الاختبار وهذا بغرض تحويل اختبارات جذر الوحدة الذاتية Tests وهو ما أطلق عليه اختبار ديكي-فوللر الموسع Augmented Dickey Fuller (ADF) Test. ويبدأ اختبار ADF بافتراض ان السلسلة الزمنية

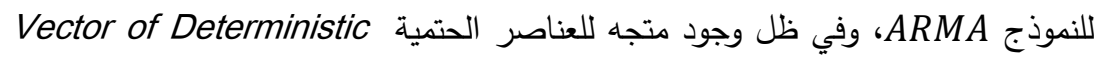
Terms استخدام 
Serial- المبطات بحيث يكون حد الخطاء $\epsilon_{t}$

. Correlation

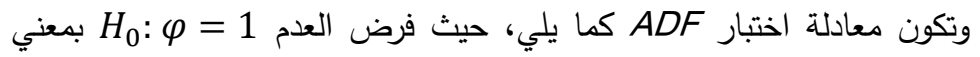

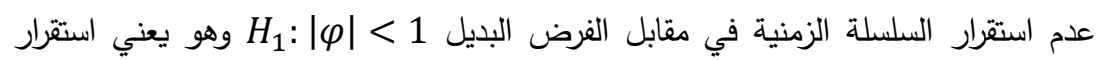

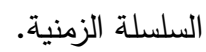

$$
Y_{t}=\beta^{\prime} \boldsymbol{D}_{t}+\varphi Y_{t-1}+\sum_{j=1}^{J} \psi_{j} \Delta Y_{t-j}+\epsilon_{t}
$$

ويمكن إعادة كتابتها في شكل الفرق الأول كما يلي، بحيث يكون البل 1 البديل 0 > 0

$$
\Delta Y_{t}=\beta^{\prime} \boldsymbol{D}_{t}+\pi Y_{t-1}+\sum_{j=1}^{J} \psi_{j} \Delta Y_{t-j}+\epsilon_{t}
$$

ومن ثم فان ADF tatistic بالنسبة لـ

$$
A D F_{n}=\frac{T(\hat{\varphi}-1)}{1-\hat{\psi}_{1}-\cdots-\hat{\psi}_{j}}
$$

قام كل من فيليبس وبيرون بتطوير عدد من اختبار جذر الوحدة، وهي التي تعرف

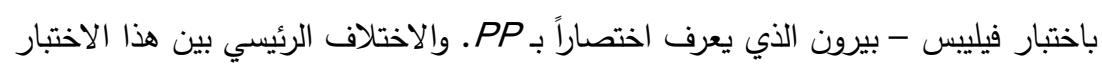

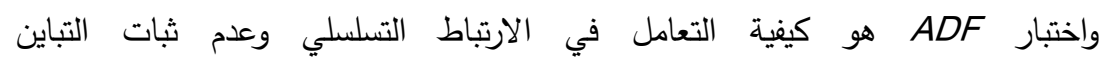

"في حالة اختيار عدد مبطأت صغير فأن الارتباط التسلسلي المتبقي في السلسلة الزمنية سوف تجعل الاختبار متحيز Bias،

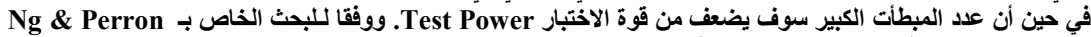

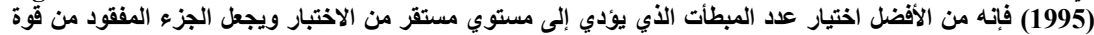

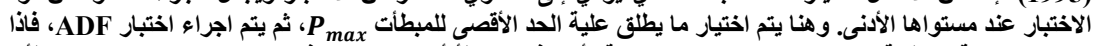

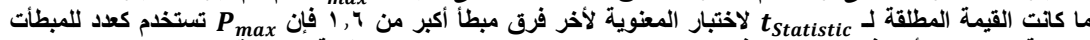

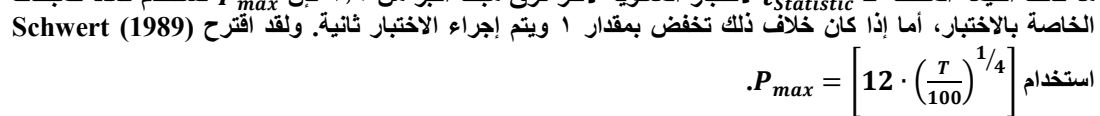


Heteroskedasticity

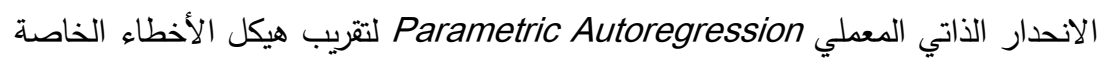
بالنموذج في شكل ARMA، إلا أن اختبار PP يتجاهل الارتباط التسلسلي في معادلة

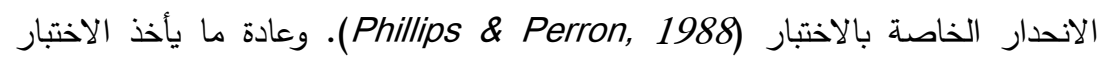

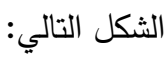

$$
\Delta Y_{t}=\beta^{\prime} \boldsymbol{D}_{t}+\pi Y_{t-1}+u_{t}
$$

ويعبر ut $u_{t}$

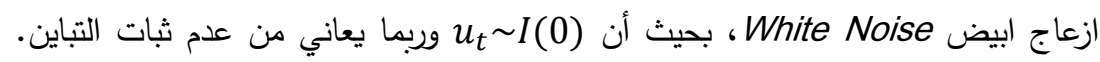

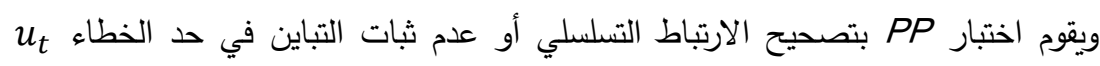
بتعديل معلمات الاختبار وهما ويتم حسابهما كما يلي: بتعدئ معنات

$$
\begin{gathered}
Z_{t}=\left(\frac{\hat{\sigma}^{2}}{\hat{\lambda}^{2}}\right) \cdot t_{\pi=0}-\frac{1}{2}\left(\frac{\hat{\lambda}^{2}-\hat{\sigma}^{2}}{\hat{\lambda}^{2}}\right) \cdot\left(\frac{T \cdot S E(\hat{\pi})}{\hat{\sigma}^{2}}\right) \\
Z_{\pi}=T \hat{\pi}-\frac{1}{2}\left(\frac{T^{2} \cdot S E(\hat{\pi})}{\hat{\sigma}^{2}}\right)\left(\hat{\lambda}^{2}-\hat{\sigma}^{2}\right)
\end{gathered}
$$

وبالنسبة لكل من $\sigma^{2}$ و فهما مقدرات متسقة Consistent Estimates لمعلمات التباين، ويحسبان كما يلي، حيث وهي OLS Regression

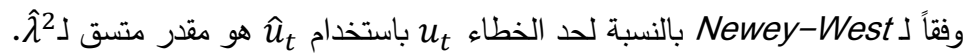

$$
\sigma^{2}=\lim _{T \rightarrow \infty} T^{-1} \sum_{t=1}^{T} E\left[u_{t}^{2}\right] \quad, \quad \lambda^{2}=\lim _{T \rightarrow \infty} \sum_{t=1}^{T} E\left[T^{-1} S_{T}^{2}\right]
$$

وفي ظل فرض العدم التوزيع التقريبي Asymptotic Distribution لاختبار ADF. ومن مزايا PP مقارنة بـ ADF 


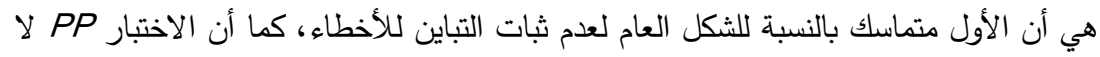

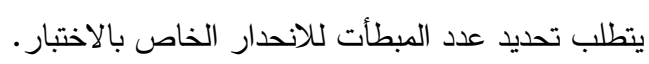

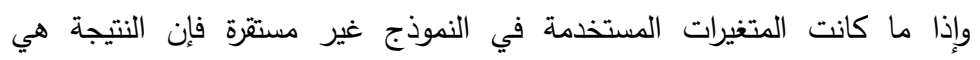

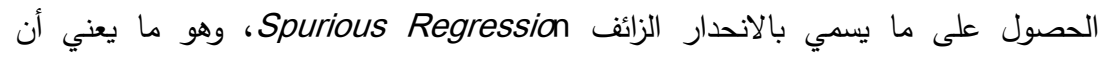
المقدرات الخاصة بالنموذج والاختبارات الإحصائية غير سليمة ومضللة ولا يمكن الاعتماد عليها Granger \& Newbold, 1974) المستقرة، وهذه الحالة مشروطة بوجود توليفة خطية من هذه المتغيرات غير المستقرة وهو يعني

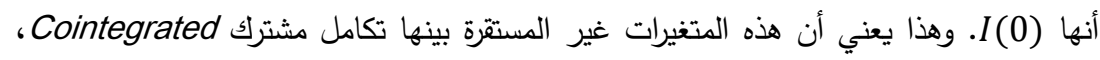
وهو ما يشير إلى وجود علاقة توازنيه طويلة الأجل بين هذه المتغيرات، حيث يوضنح

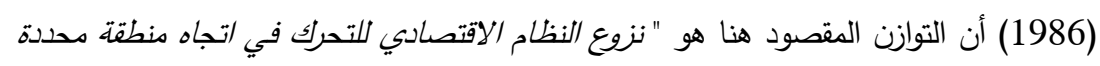

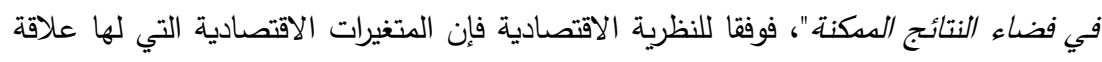

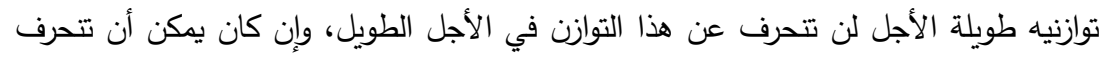

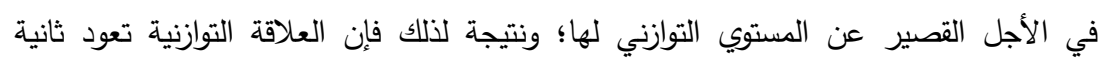

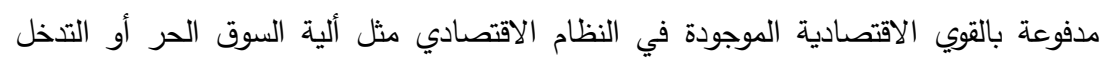
ويعرف Granger (1981) التكامل المشترك بين المتغيرات في حالة أن هناك الحكومي.

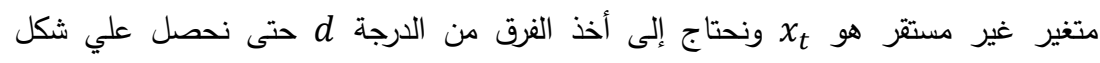

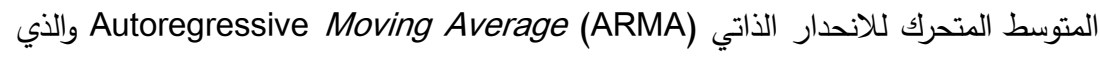

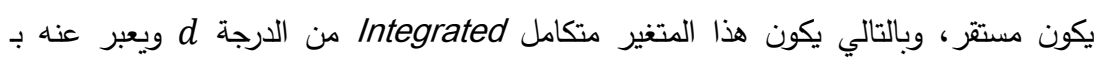

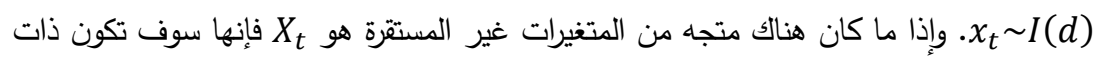
تكامل مشترك من الدرجة d, $b$ وتكتب (d)

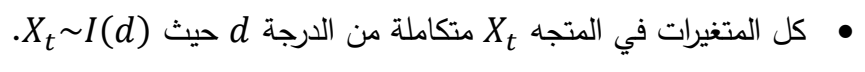

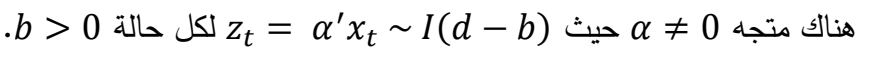

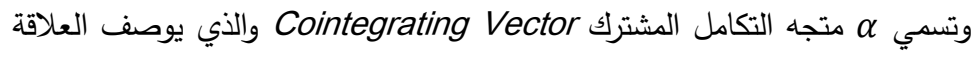
الثابتة طويلة الأجل، وإذا ما كان عدد المتغيرات في

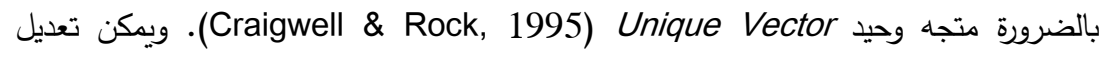


نموذج ADL لكي يصبح نموذج Error-Correction Model (ECM) وهو ما يعرف بنموذج انجل-جرانجر Engle-Granger Two-Step Model، بحديث يأخذ الثكل التالي: $\Delta Y_{t}=\alpha+\sum_{j=1}^{J} \xi_{j} \Delta Y_{t-j}+\sum_{i=1}^{N} \sum_{k=0}^{K} \omega_{i, k} \Delta \boldsymbol{X}_{i, t-k}+\Psi\left(Y_{t-1}-\alpha_{0}-\sum \beta_{i} \boldsymbol{X}_{i, t-1}\right)+\varepsilon_{t}$

حيث عنصر تصحيح الخطاء هو (الفير التكامل المشترك بين المتغير التابع والمتغيرات المستقلة، ويشير إلى العلاقة طويلة الأجل بين التين

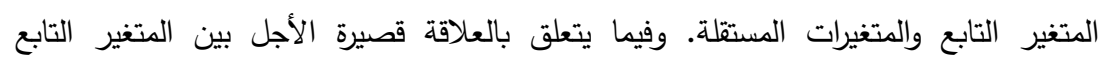
والمتغيرات المستقلة فهي معبر عنها بتجه المعلمات التعديل (تصحيح الخطاء) والتي يجب أن تكون سالبة القيمة وكذلك 1 > | النظام إلى التوازن في الأجل الطويل. ^. بيانات الاراسة ومصادرها إن البيانات المستخدمة في هذا البحث فهي بيانات سلسلة زمنية سنوية للمتغيرات

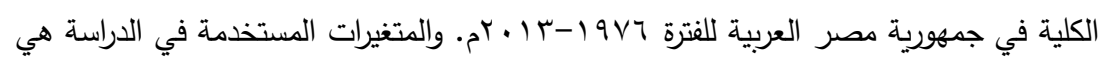

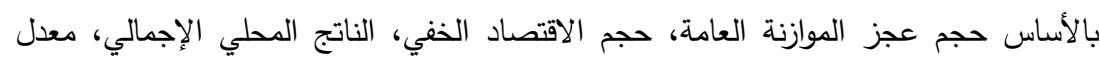

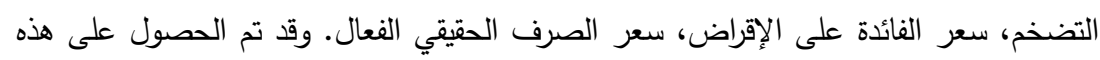

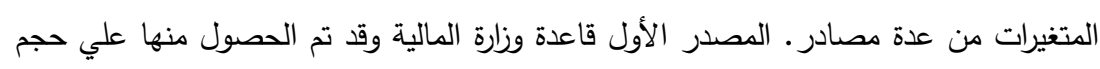

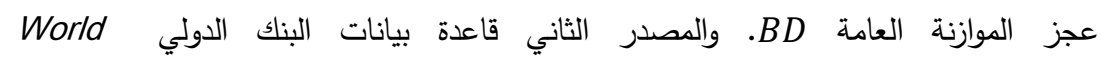
Development Indicators

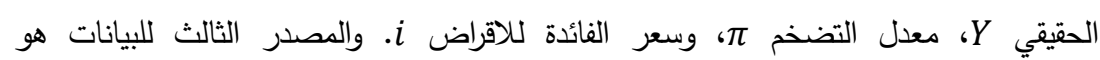
Bruegel Database

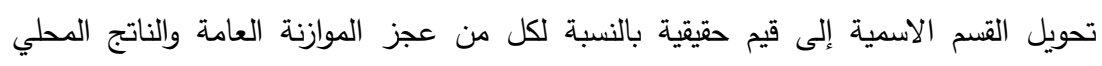

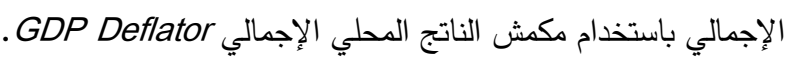

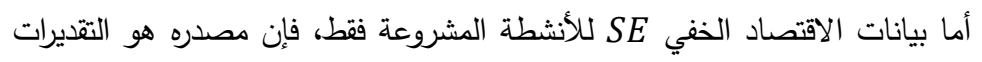
التي قام بها كل من Maiedrich Schneider Massan في دراستهم التي قاما فيها

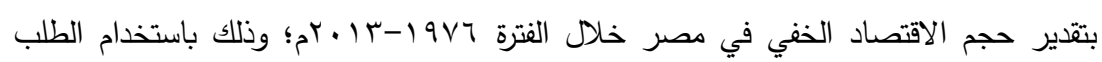


على العملة CDA وطريقة المؤشرات المتعددة والمسبيات المتعددة MIMIC، ويتضح من الشكل

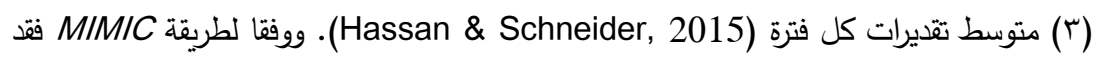
كانت المتغيرات المسببة لتنامي الاقتصاد الخفي هي: إجمالي الضرائب كنسبة من GDP، الجودة المؤسسية، القيمة المضافة في قطاع الزراعة كنسبة من GDP، معدل البطالة، نسبة التوظيف الذاتي لقوة العمل. أما المؤشرات المتأثرة بتتامي الاقتصاد الخفي هي: الناتج المحلي الإجمالي الحقيقي، النمو في عرض النقود، نسبة التوظف الكلي لعدد السكان. وفي نموذج كانت المتغيرات المستخدمة في تقدير حجم الاقتصاد الخفي هي: حجم العملة المتداولة، الناتج المحلي الإجمالي الحقيقي، العبء الضريبي الكلي، حجم التوظيف في القطاع العام كنسبة من إجمالي قوة العمل، معدل التوظف الذاتي، سعر الفائدة الاسمي على الودائع.

(r)

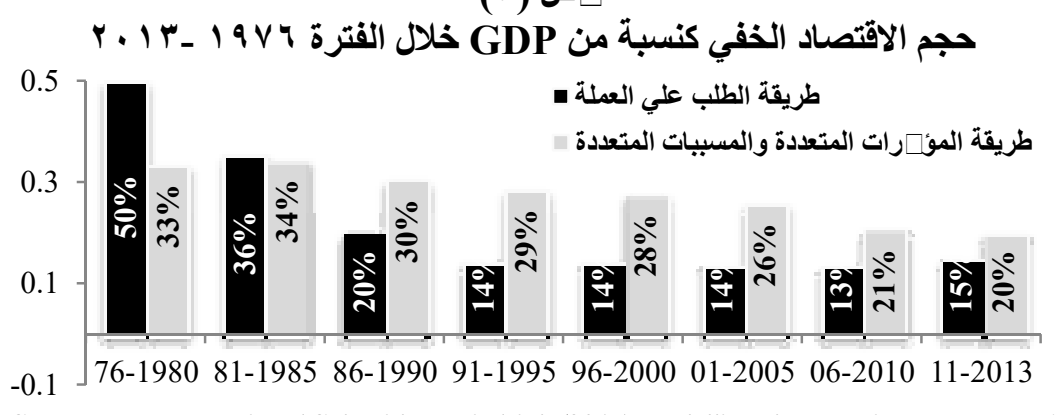

Source: Hassan, Mai and Schneider, Friedrich (2015) Modelling the Egyptian Economy: A currency Demand and A MIMIC Model Approach, A Preliminary Draft, Center of Near and Middle Eastern Studies, Department of Middle East Economics, Germany.

ونظرا لوجود تقديران لحجم الاقتصاد الخفي في الاقتصاد المصري وفقا للدراسة التي

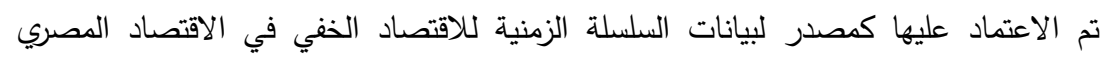
(Hassan \& Schneider, 2015). فقد تم رسم العلاقة ما بين نسبة عجز الموازنة العامة كنسبة من GDP وحجم الاقتصاد الخفي كنسبة من GDP لمعرفة إيهما أقرب للتتبؤ باتجاه عجز الموازنة العامة. ومن الشكل (乏) يتضح أن طريقة CDA تعطي تقديرات أكثر توافقا مع عجز الموازنة العامة، كما سيؤكد النموذج المقدر لاحقا. 
(

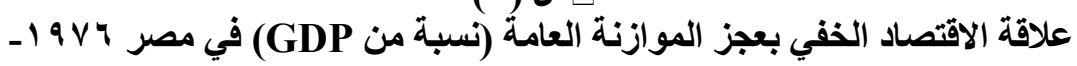
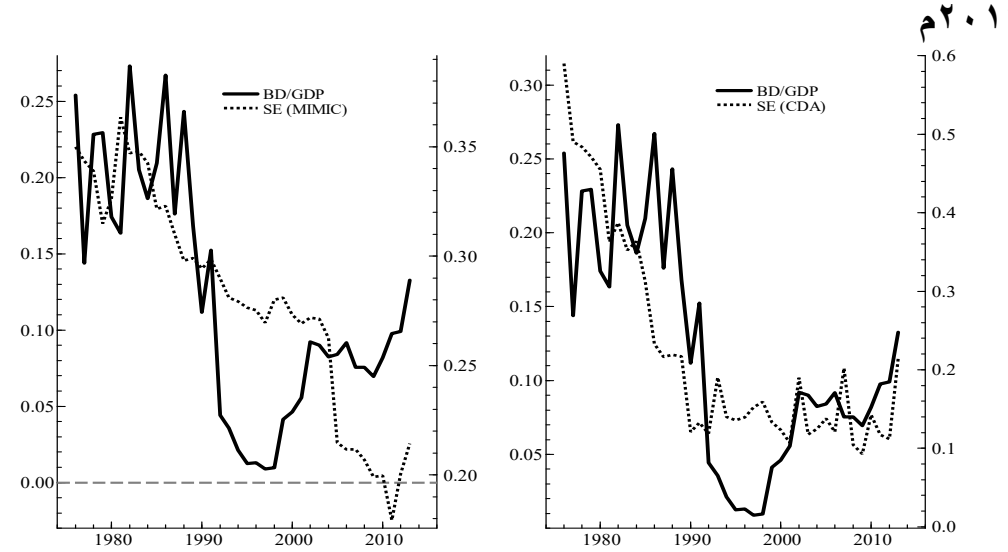

يظهر الجدول (ع) متوسط قيم المتغيرات في الفترات الزمنية (كل خمس

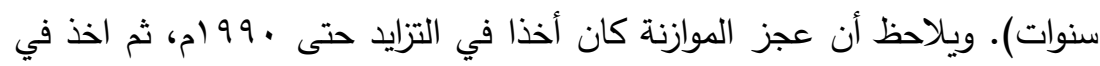
التراجع كنتيجة لبرنامج الإصلاح الاقتصادي ERSAP، ثم تزايد ثانية مع بداية القرن الواحد والعشرون. أما الناتج المحلي الإجمالي الحقيقي فقد كان أخذا في التزايد بشكل مستمر طيلة الفترة محل الدراسة. أما الاقتصاد الخفي فقد كان متوسطة في في الإني

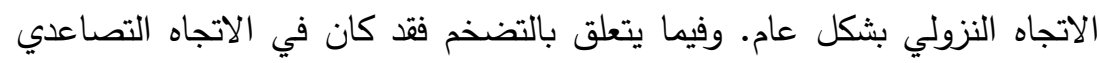

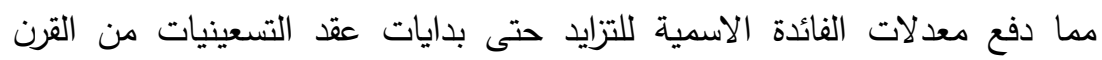

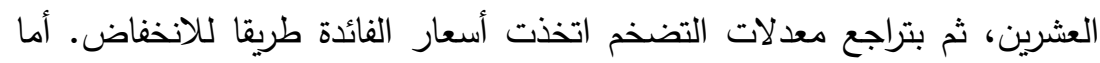
سعر الصرف الحقيقي الفعال فقد كان عرضة للتذبذب نتيجة العديد من التطورات

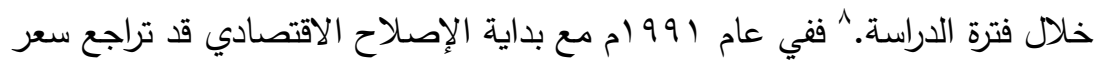

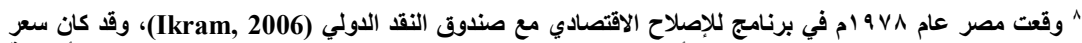

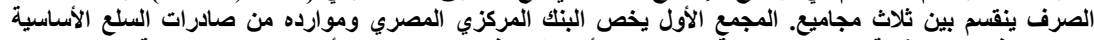

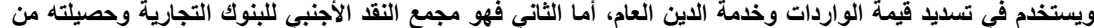

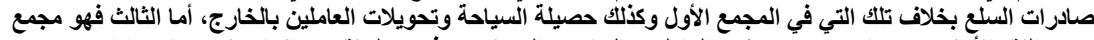

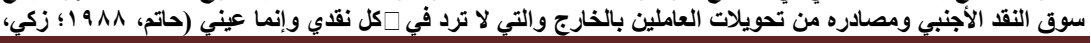

Yos

المجلة العلمية للاقتصاد والتجارة 


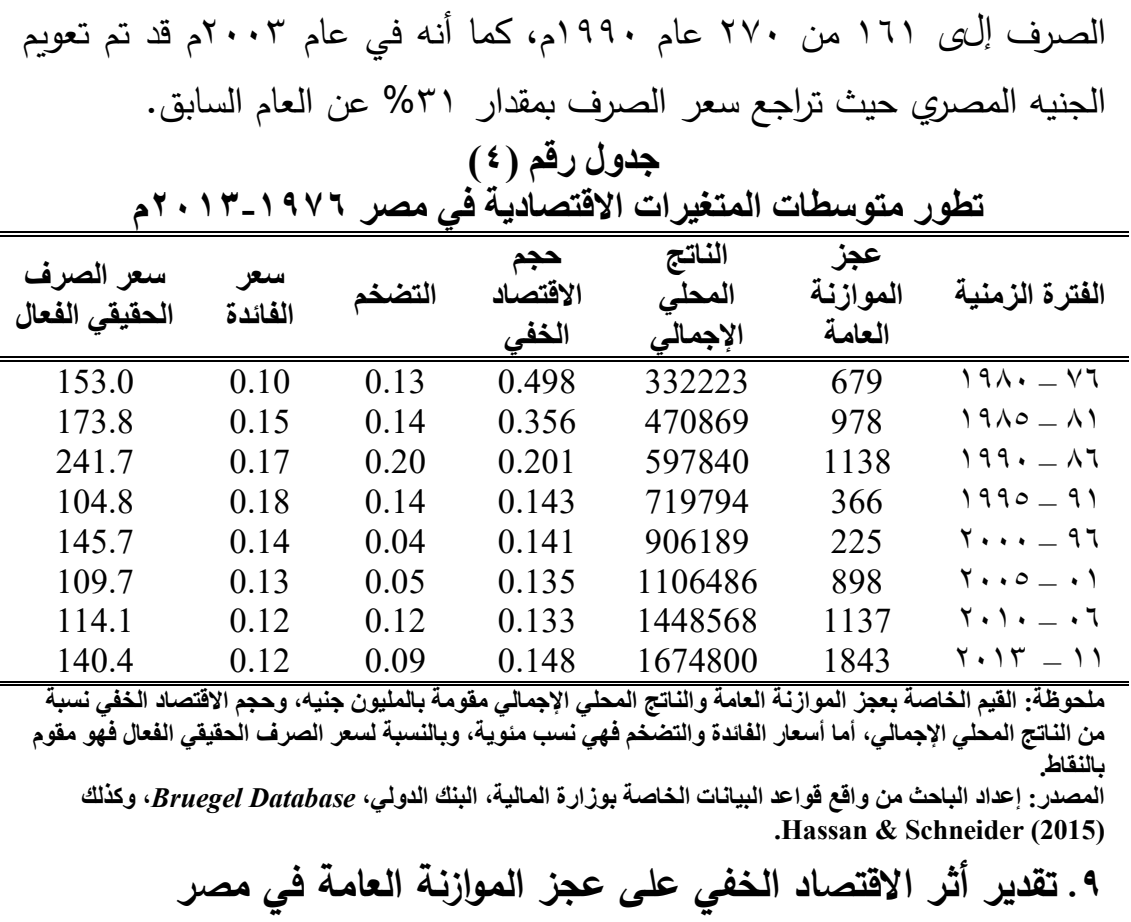

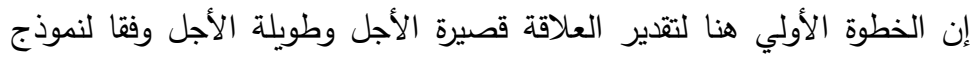

Engle-Granger Two-Step جذور الوحدة (PP \& ADF) إلى أن المتغيرات المستخدمة كلها غير مستقرة في المستوي

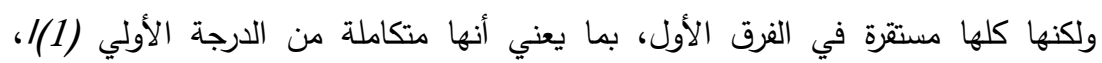

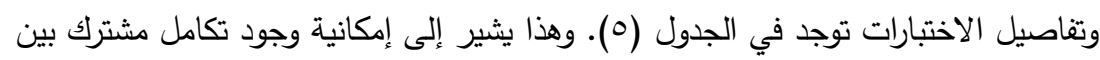
هذه المتغيرات ذات السلاسل الزمنية غير المستقرة. 


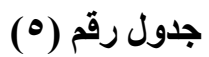

\begin{tabular}{|c|c|c|c|c|}
\hline \multicolumn{2}{|c|}{ PP-test } & \multicolumn{2}{|c|}{$A D F$-test } & \multirow{2}{*}{ المتغير } \\
\hline الفروق & المستوي & الفروق & المستوي & \\
\hline$-6.05^{*}$ & -1.55 & $-5.95 *$ & -2.500 & $B D$ \\
\hline$-8.00 *$ & -1.69 & $-7.82 *$ & -2.02 & $S E$ \\
\hline$-4.82 *$ & $-4.96^{*}$ & $-4.75^{*}$ & -2.17 & $y$ \\
\hline$-11.05^{*}$ & -2.38 & $-10.79 *$ & -2.24 & $\pi$ \\
\hline $2.95^{*}$ & -2.34 & $-2.84^{*}$ & -2.67 & $i^{L}$ \\
\hline$-4.19 *$ & -2.35 & $-4.37^{*}$ & -2.06 & reer \\
\hline
\end{tabular}

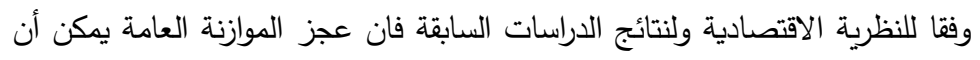

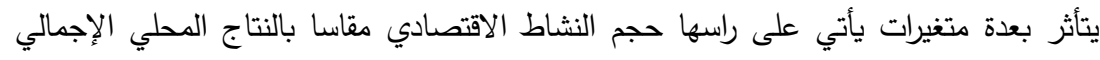

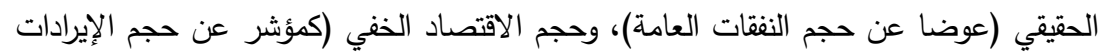

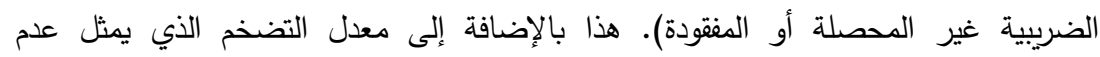

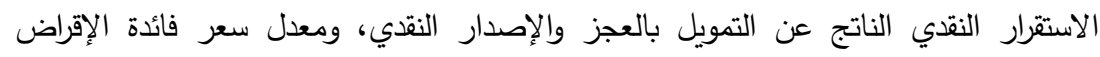

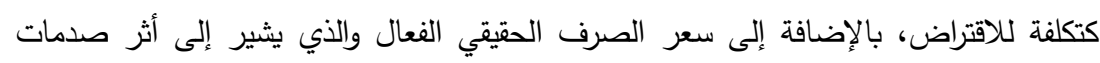

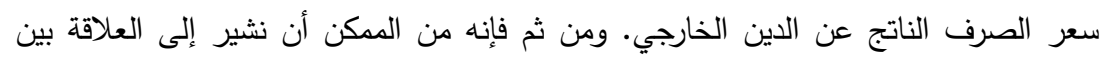

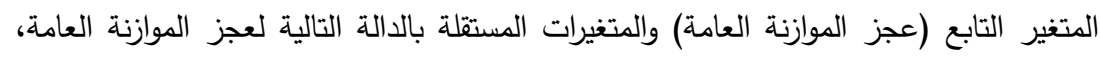

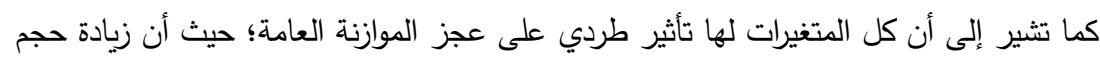

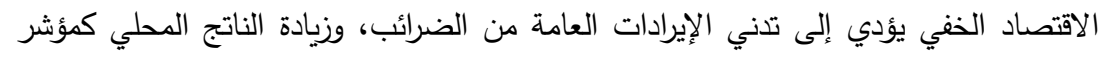

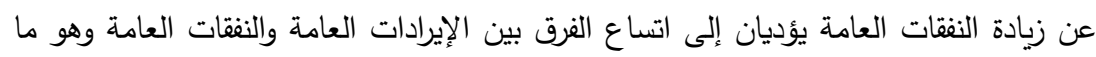

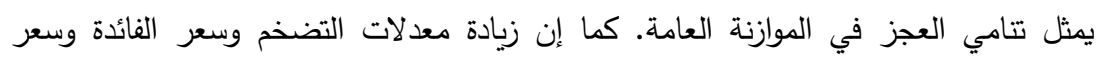

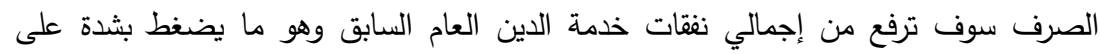
الموازنة العامة ومن ثم يتسع حجم العجز في الموازنة العامة.

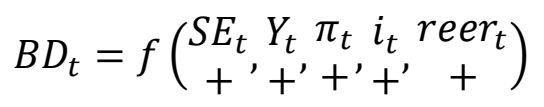


وبافتراض وجود العلاقة الخطية بين المتغيرات، وذلك بعد تحويل عجز الموازنة

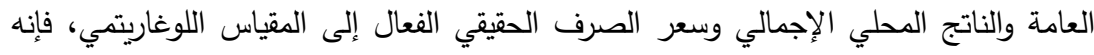
يمكن التعبير عن نموذج الاتحدار المثل للعلاقة كما يلي:

$$
\widehat{B D}_{t}=\widehat{\boldsymbol{\alpha}}+\widehat{\boldsymbol{\beta}}^{\prime}\left[\begin{array}{c}
S E_{t} \\
Y_{t} \\
\pi_{t} \\
i_{t} \\
\text { reer }_{t}
\end{array}\right]+\boldsymbol{\epsilon}_{t}
$$

وتكون معادلة الانحدار المقدرة وفقا للنموذج السابق كما يلي حيث المعلمات المقدرة

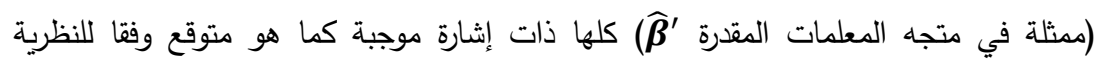
الاقتصادية والدراسات السابقة. وبالنظر إلى اختبارات جودة التوفيق فإنها كلها جيدة.

$$
\widehat{B D}_{t}=-31.17+7.81 S E_{t}+2.36 Y_{t}+6.11 \pi_{t}+6.19 i_{t}+0.48 \text { reer }_{t}
$$

$T=38 R^{2}=0.46 \quad R_{a d j}^{2}=0.38 \quad R S S=14.97 \quad \sigma=0.104 \quad F=5.55[0.00]^{9}$ وبالحصول على أخطاء التقدير من النموذج السابق تم اختبارها للتأكد من إنها مستقرة

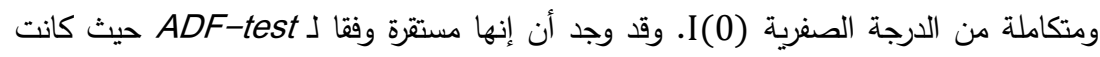

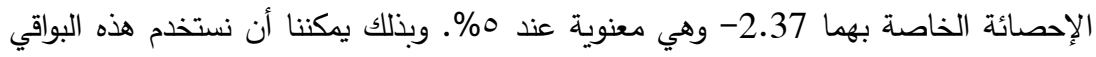
بعد إبطائها بفترة واحدة حيث سوف تعبر عن عنصر تصحيح الخطاء للرجوع إلى التوازن ويشار

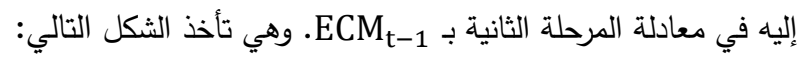

$$
E C M_{t-1}=\boldsymbol{\epsilon}_{t-1}=\widehat{B D}_{t-1}-\widehat{\boldsymbol{\alpha}}-\widehat{\boldsymbol{\beta}}^{\prime}\left[\begin{array}{c}
S E_{t-1} \\
Y_{t-1} \\
\pi_{t-1} \\
i_{t-1} \\
\text { reer }_{t-1}
\end{array}\right]
$$

ويتم تقدير معادلة المرحلة الثانية من نموذج Engle-Granger والتي تمثل العلاقة

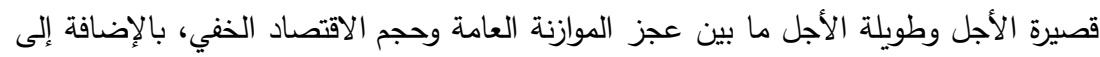

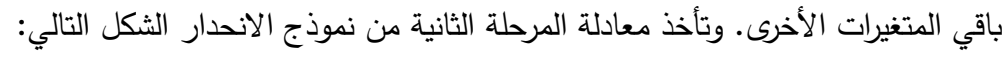

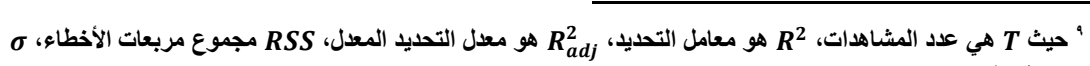

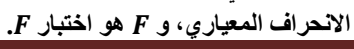




$$
\Delta \widehat{B D}_{t}=\widehat{\boldsymbol{\alpha}}+\sum_{j=1}^{J} \widehat{\xi}_{j}^{\prime} \Delta B D_{t-j}+\sum_{j=\mathbf{0}}^{J} \widehat{\boldsymbol{\omega}}^{\prime}\left[\begin{array}{c}
\Delta S E_{t-j} \\
\Delta Y_{t-j} \\
\Delta \pi_{t-j} \\
\Delta i_{t-j} \\
\Delta r e e r_{t-j}
\end{array}\right]+\widehat{\mathbf{\Psi}} E C M_{t-1}+\widehat{\boldsymbol{\delta}}^{\prime}\left[\begin{array}{l}
\text { Dum } 95_{t} \\
\text { Dum99t }
\end{array}\right]+\boldsymbol{\varepsilon}_{t}
$$

والتغيرات في المتغيرات التابعة تفسر Yr\% من إجمالي التغيرات الحادثة في عجز

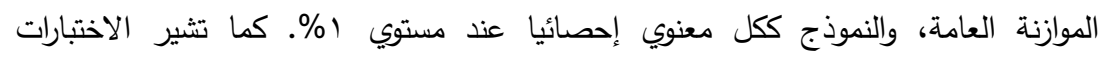

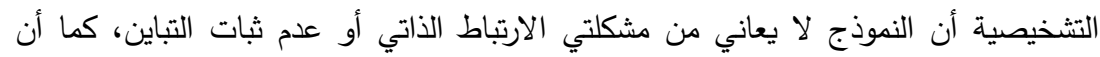

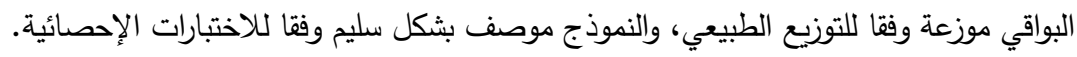

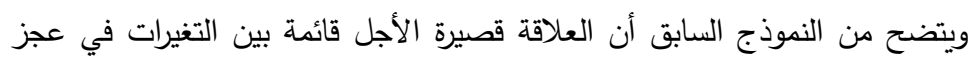

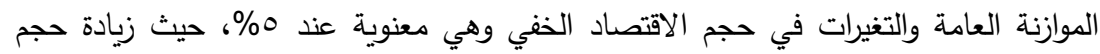

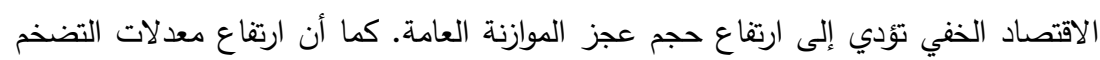

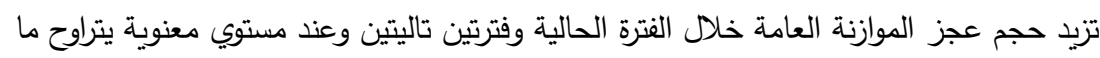

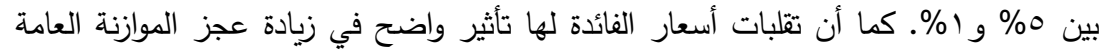

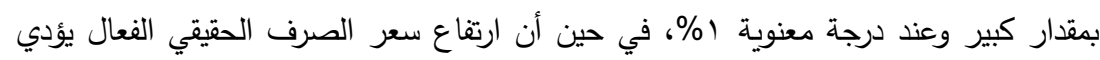

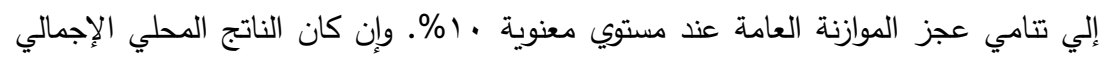

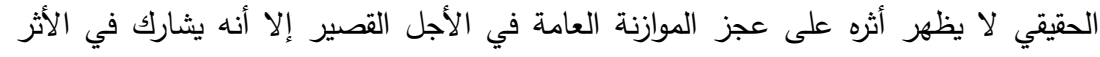

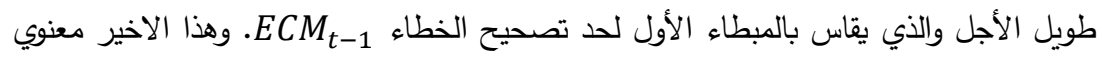

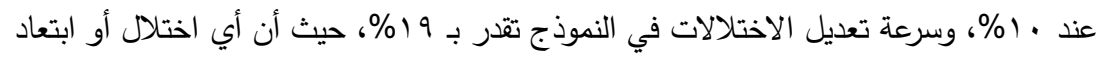

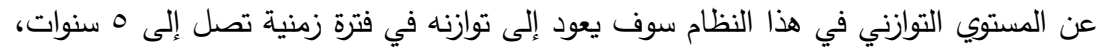

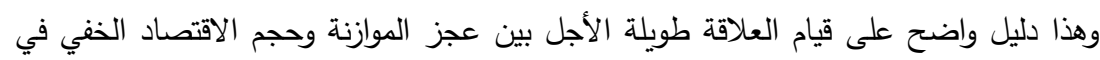

$$
\Delta B D_{t}=\underset{0.05}{0.98]}+2.25 \Delta S E_{t}+2.86 \Delta \pi_{t}+2.39 \Delta \pi_{t-2}+17.89 \Delta i_{t}
$$

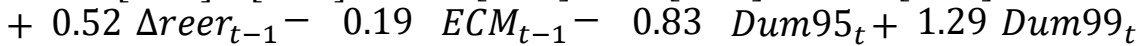

$$
\begin{aligned}
& \text { [1.79] } \\
& \text { [-1.76] } \\
& {[-2.75]} \\
& \text { [4.24] } \\
& R_{\text {adj }}^{2}=0.63 \quad R S S=1.66 \quad \sigma=0.26 \quad F=7.86[0.00] \quad R^{2}=0.72
\end{aligned}
$$




$$
\begin{array}{rll}
F_{\text {het }}[12,18]=0.42[0.94] & F_{\text {arch }}[1,31]=0.88[0.36] & F_{\text {ar }}[2,22]=0.48[0.62] \\
F_{\text {reset }}[2,22]=4.60[0.02]^{\prime} \cdot & \chi_{\text {nor }}^{2}[2]=3.14[0.21]
\end{array}
$$

ولقد تم إضافة متغيرين صوريين هما Dum95 و

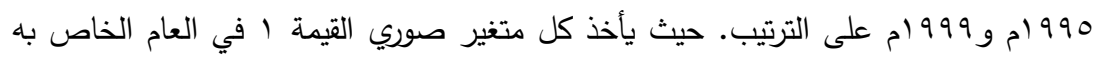
(990 ام أو 999 (م))، ويأخذ القيمة · في باقي سنوات العينة. ويتضح أن كلا المتغيرين معنوي

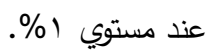

والمتغير الصوري Dum95 يظهر تاثيرة السالب علي عجز الموازنة العامة؛ حيث هناك العديد من الإصلاحات الاقتصادية التي قامت بها الحكومة المصرية في عام 990 ام وكان لها أثر واضح في خفض عجز الموازنة العامة في هذا العام." وكان علي رأس هذه الإصلاحات زيادة أسعار الطاقة (البترول والكهرباء) بخفض الداعم عليها، تطبيق ضريبة القيمة المضافة متعددة المراحل وتوسيع نطاق القاعدة الضريبية، تحويل هب\% من أصول الشركات العامة إلى القطاع الخاص، ومحاولة استكمال بيع أحد بنوك القطاع العام وبيع حصة القطاع العام في بنوك الاستثمار المشترك، وكذلك إلغاء التعريفة الجمركية التفضيلية لقطاع الصحة

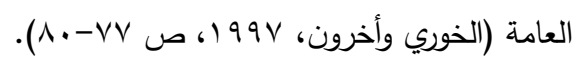

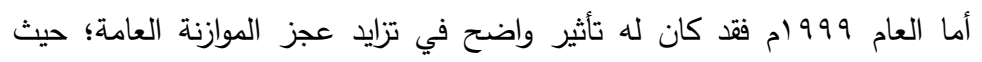

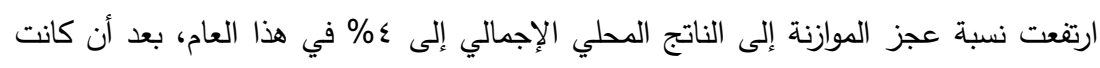

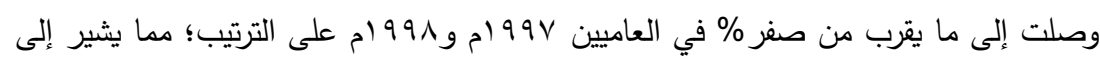
التغير الحاد في مسار عجز الموازنة العامة في مصر، نظرا لتضافر العديد من الأسباب، منها: ظهور أثار أزمة جنوب شرق أسيا في الأعوام التالية للعام 99 ا9 ام، وارتفاع أسعار البترول عالميا نتيجة اتفاق دول الأوبك مع دول الخليج العربي علي تخفيض الإنتاج حتي وصل السعر

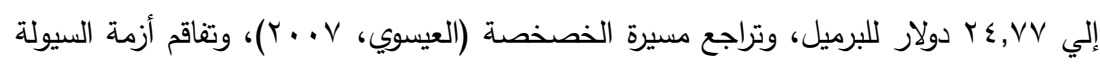
المحلية في العام 999 ام (الرفاعي، .... ب)، هذا بالإضافة إلي تفاقم أزمة التعثر المصرفي

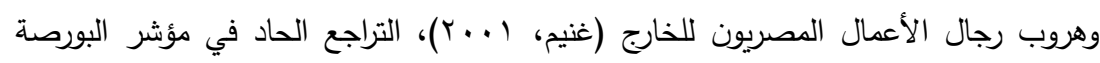

. ' التوزيع الطبيعي،

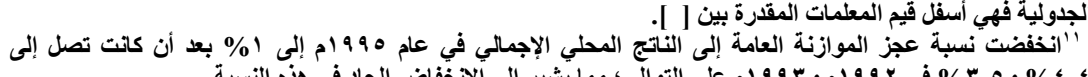

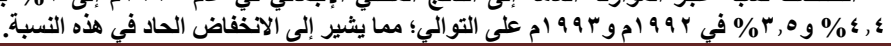

roq

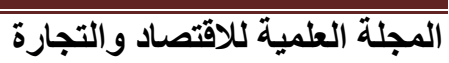


المصرية نتيجة تفاقم أثار أزمة جنوب شرق أسيا (Abdelkader, 2017b)، هذا بالإضافة إلي

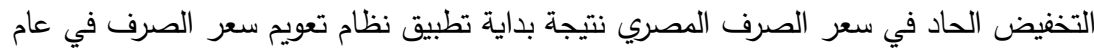
.(Abdelkader, 2017a) م1999 إن استقرار العلاقة المقدرة خلال الفترة الزمنية محل الدراسة أمر هام؛ ولذلك فقد تم

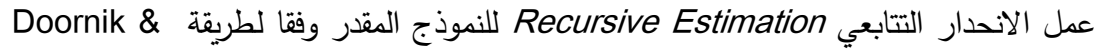
Hendry (2009) نظراً للتحولات في النظام الاقتصادي المصري متمثلا في الإصلاح الاقتصادي ERSAP. والثكل (0) يظهر استقرار المعلمات المقدرة. والثكل (؟) يظهر استقرار

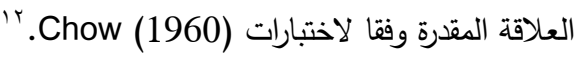
(•)

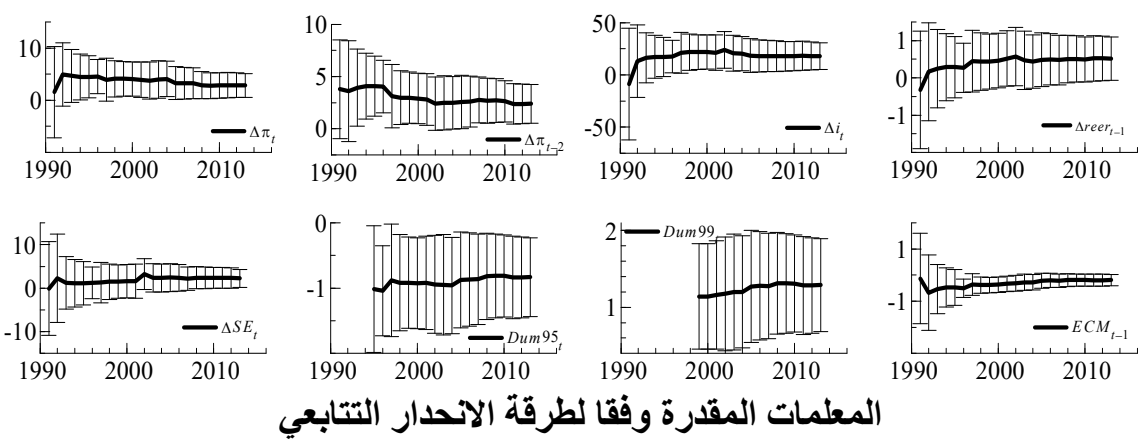

بتم الرجوع إلى (2009) Doornik \& Hendry واستخدام المعادلات التالية في اختبارات Chow كما يلي:

1-Step Chow Test

Break-Point Chow Test

Forecast Chow Test
$\frac{\left(R S S_{t}-R S S_{t-1}\right)(t-k-1)}{R S S_{t-1}}=\frac{v_{t}^{2} / \omega_{t}}{\widehat{\sigma}_{t-1}^{2}}$

$\frac{\left(R S S_{T}-R S S_{t-1}\right)(t-k-1)}{R S S_{t-1}(T-t+1)}=\frac{\frac{1}{T-t+1} \sum_{m}^{T} v_{m}^{2} / \omega_{t}}{\hat{\sigma}_{t-1}^{2}}$

$\frac{\left(R_{S S}-R S S_{M-1}\right)(M-k-1)}{R S S_{M-1}(t-M+1)}$

r.

المجلة العلمية للاقتصاد والتجارة 


\section{(7) (7)}

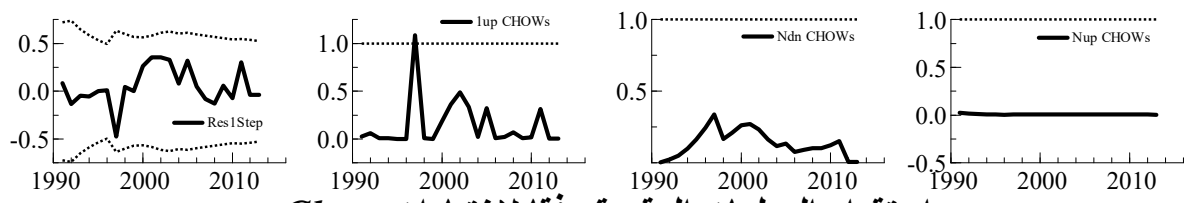

Chow استقرار المعلمات المقدرة وفقا لاختبارات

إن القدرة على التتبؤ بمستقبل عجز الموازنة العامة في مصر في ظل وجود

الاقتصاد الخفي، تم اختباره للتأكد مما إذا كان النموذج المقدر يصلح للتنبؤ أم لا. والقيم المقدرة لا تختلف عن القيم الفعلية لعجز الموازنة العامة وان هناك فارق في العام II لـrم نظرا لنشوب الثرة المصرية فيما يعرف بالربيع العربي. والشكل (V) يوضح التبؤ داخل العينة In-Sample Forecast لعجز الموازنة العامة، ويتضح من الشكل أن النموذج المقدر له قدرة تتبؤيه عالية.

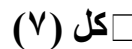

القدرة التتبؤية لنموذج عجز الموازنة والاقتصاد الخفي

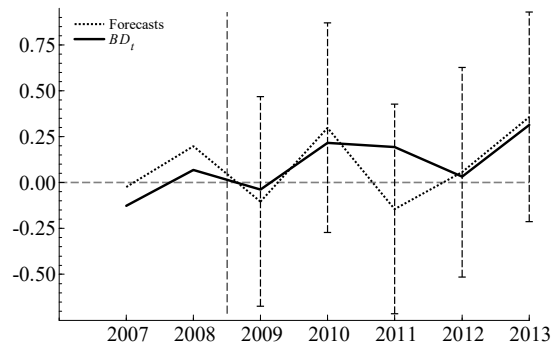

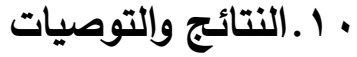

إن أهم نتائج هذا البحث هي قبول فرض الدراسة وهو لإن تنزيد حجم الاقتصاد الخفي يؤلي إلى تنايل عجز الموازنتة العامة للدولتة في مصر خلال فترة الدراسة "، وقد تم إثبات هذا الفرض حيث أن النموذج المقدر اثبت اقتصاديا وإحصائيا وجود علاقة طردية قوية 
في الأجل القصير والطويل ما بين عجز الموازنة العامة وحجم الاقتصاد الخفي (للأنشطة

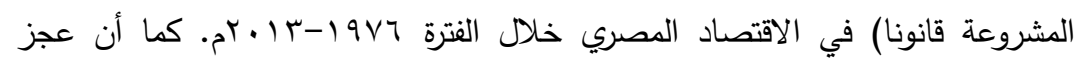

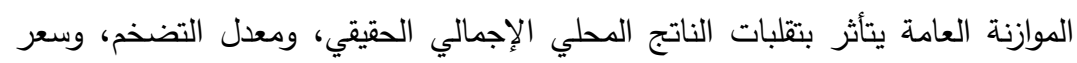

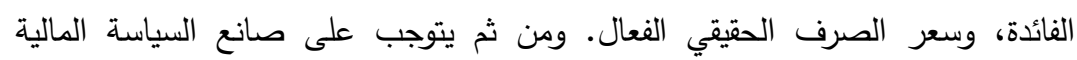

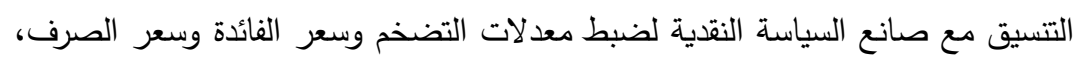

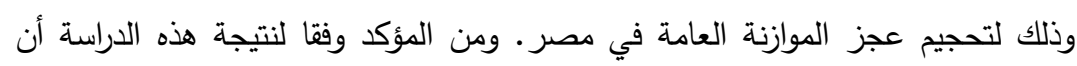

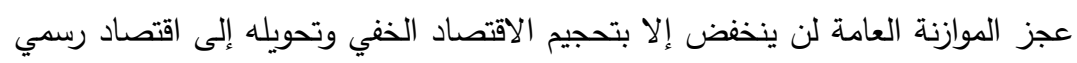
وفقا للتوصيات التالية.

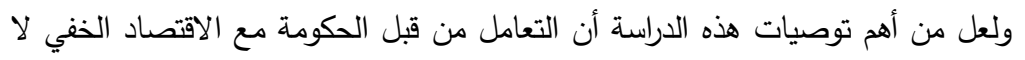

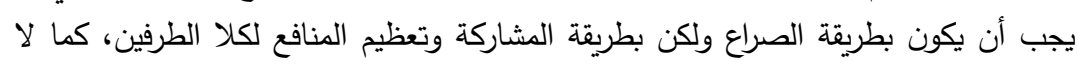

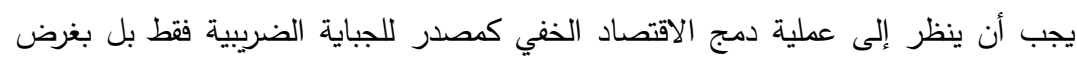

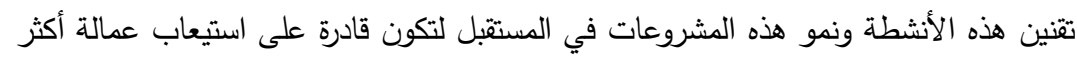

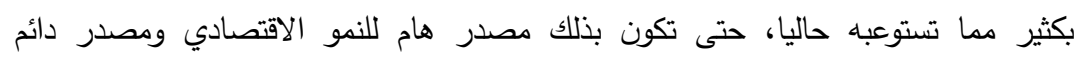

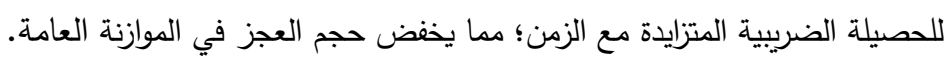

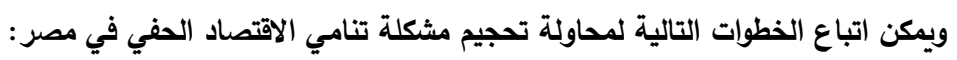

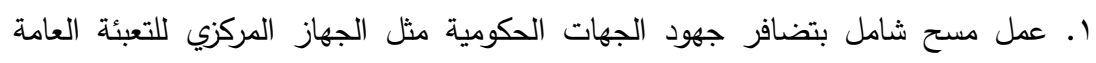

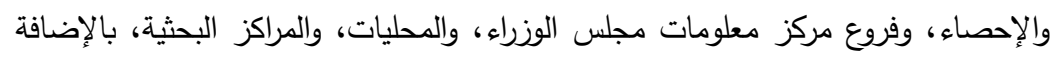

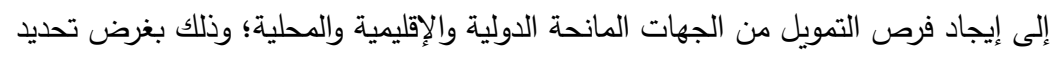

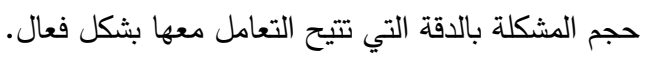

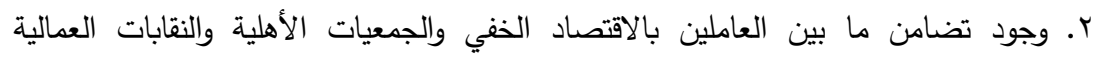

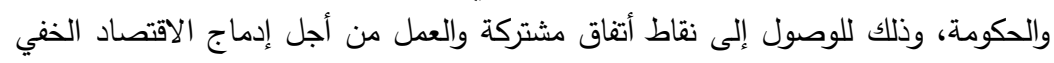

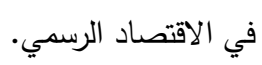
r. إحياء رأس المال الميت (ويقصد به الأصول غير المسجلة بصكوك ملكية)، وذلك بتيسير

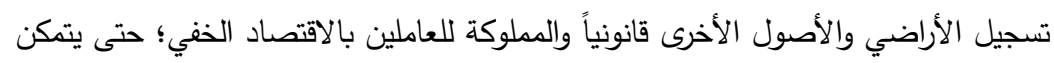

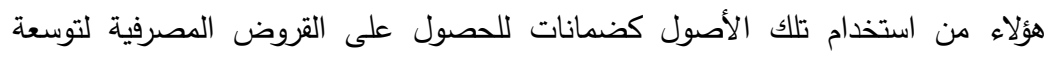
أنشطتهم وإدماجهم في الاقتصاد الرسمي. 
ع. توفير مصادر تمويل مصرفية للأنثطة التي تتحول من الاقتصاد الخفي إلى الاقتصاد

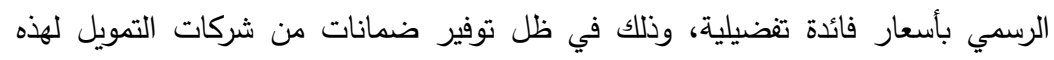

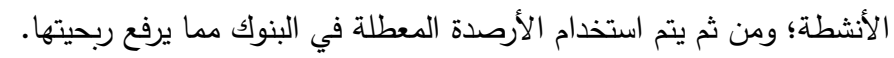

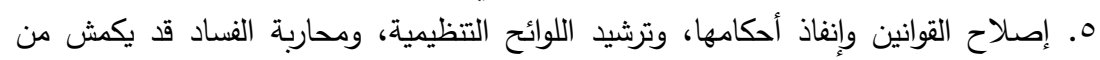
حجم الاقتصاد الخفي.

7 ا تبسيط الإجراءات وتفعيل منفذ الخدمة الواحد الذي يجمع كل الخدمات التي تقدمها الوزارات

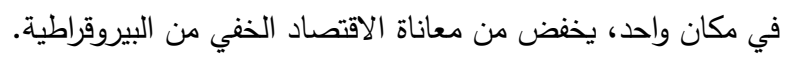
V. ضبط فساد المحليات، وتراخيص البناء، واشغالات الطرق، والباعة الجائلين، واستخراج

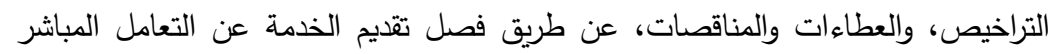
بين المواطنين والموظفين بواسطة الحكومة الإكترونية وتقديم الخدمات الحكومية إلكترونياً مما يكمش من الفساد ويقلص من الاقتصاد الخفي. ^. إتاحة وتحرير الأنشطة الاقتصادية مثل تحرير سوق العمل قد يقلص من حجم العمالة في الاقتصاد الخفي. 9. إجراء تخفيضات كبيرة في معدلات الضرائب لن يؤدي إلى تخفيض كبير في حجم الاقتصاد الفياد الخفي، لكنه قد يثبت حجمه. كما أن تدقيق الحسابات الضريبية، وجعلها أكثر دورية وتثديد الفيد

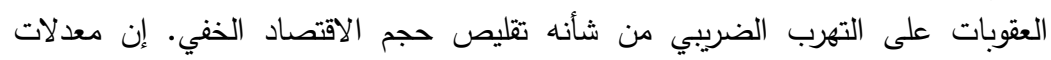

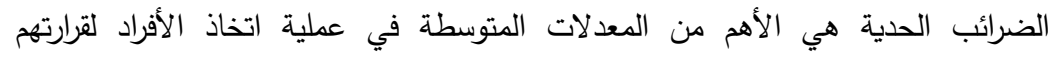

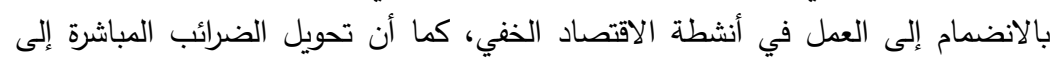
غير مباشرة لن يحسن وضع الامتثال الضريبي. •l.

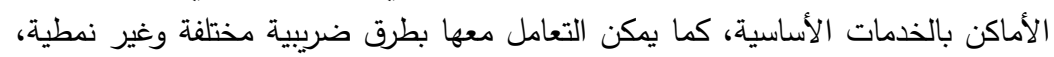

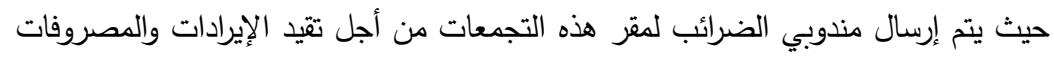

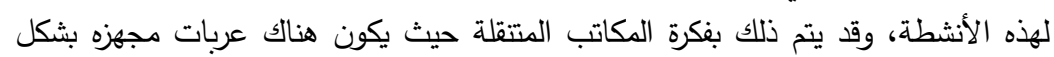
مكتب به حاسب إلى مرتبط بالأنترنت والجهات الحومية، وبالتالي تتنقل الخدمة الحكومية لالتيات إلى هؤلاء في أماكن عملهم.

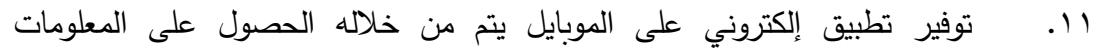
الأساسية لتحويل الأنشطة من الاقتصاد الخفي إلى الاقتصاد الرسمي، كما يتم من خلالدا التقديم للحصول على الخدمات والتراخيص، وإرسال الثكاوى، وسداد الرسوم والضرائب. 


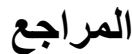

أولا المراجع العربية:

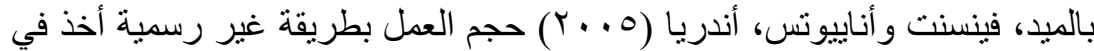

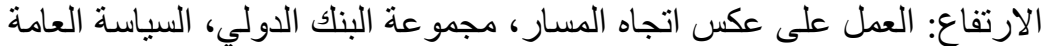

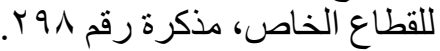

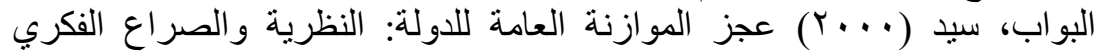

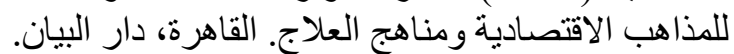

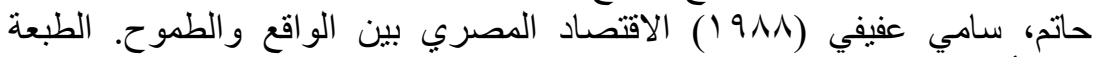
الأولي، القاهرة، دار النشر الدولية.

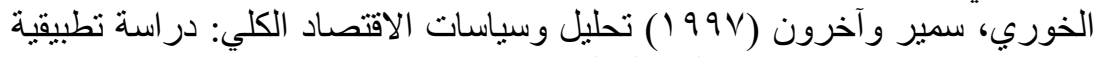
عن مصر. معهد صندوق النقد الدولي، و اشنطن.

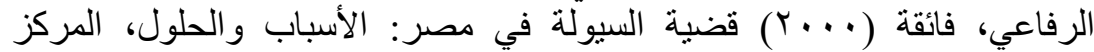

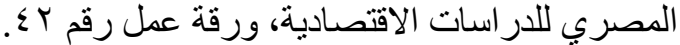

زكي، رمزي (9101) بحوث في ديون مصر الخارجية. الطبعة الأولي، القاهرة،

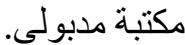

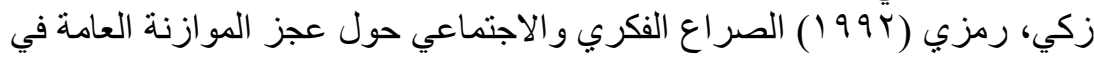

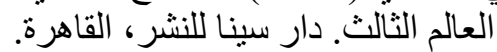

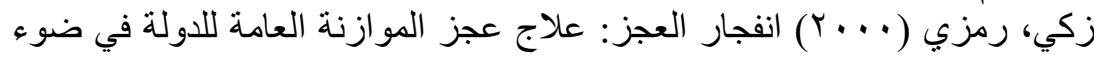

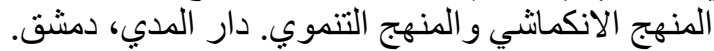

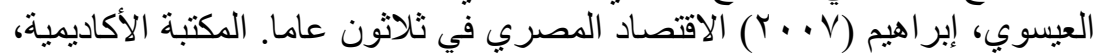

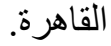

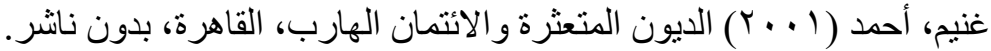

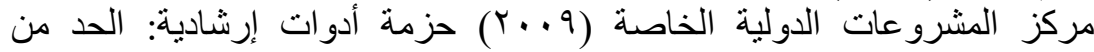

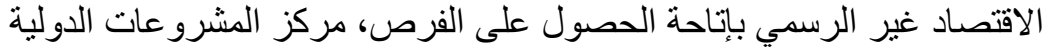

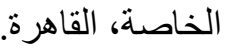
ثانيا المراجع الأجنية: الخية:

Abdelkader, Hossam E. M. (2017a) Investigating the Transmission Mechanism of Monetary Policy in Egypt, LAP Lambert Academic Publishing, Germany.

Abdelkader, Hossam E. M. (2017b) The Story of Egyptian Economy between Two Revolutions. LAP Lambert Academic Publishing, Germany.

Y $₹$

المجلة العلمية للاقتصاد والتجارة 
Alm, J., \& Embaye, A. (2013) Using dynamic panel methods to estimate shadow economies around the world, 1984-2006. Public Finance Review, 41(5), 510-543.

Chow, G. C. (1960) Tests of Equality between Sets of Coefficients in Two Linear Regressions, Econometrica, 28(3), 591-605 .

Çiçek, D., \& Elgin, C. (2011) Cyclicality of fiscal policy and the shadow economy. Empirical Economics, 41(3), 725-737.

Craigwell, R.C., Rock, L.L., (1995) An Aggregate Consumption Function for Canada: A Cointegration Approach, Applied Economics 27, 239-249.

Doornik, J. A., \& Hendry, D. F. (2009) Empirical Econometric Modelling PcGive 13: Volume I (Vol. Volume I). London: Timberlake Consultants Ltd.

Enste, Dominik H. (2015) The Shadow Economy in Industrial Countries, IZA World of Labor, No. 127, 1-10.

Granger, C. W. J., \& Newbold, P. (1974) Spurious Regressions in Econometrics, Journal of Econometrics, 2(2), 111-120 .

Granger, C.W.J., (1981) Some Properties of Time Series Data and Their Use in Econometric Model Specification, Journal of Econometrics 16, 121-130.

Granger, C.W.J., (1986) Developments in the Study of Cointegrated Economic Variables. Oxford Bulletin of Economics and Statistics $48,213-228$.

Hassan, Mai and Schneider, Friedrich (2015) Modelling the Egyptian Economy: A currency Demand and A MIMIC Model Approach, A Preliminary Draft, Center of Near and Middle Eastern Studies, Department of Middle East Economics, Germany.

Hassan, Mai and Schneider, Friedrich (2015) Modelling the Egyptian Economy: A currency Demand and A MIMIC Model Approach, Journal of Economic and Political Economy, Vol 3(2), 309-339.

Ikram, K. (2006) The Egyptian Economy 1952-2000: Performance, Policies, and Issues, $1^{\text {st }}$ Ed, London, Routledge.

Kassem, Taha (2014) Formalizing the Informal Economy: A Required State Regulatory and Institutional Approach: Egypt as a Case Study, International Journal of Humanities and Social Science, Vol. 4, no. 1, January, P. 27-48.

Loayza, $N . V$. (1996) The economics of the informal sector: a simple model and some empirical evidence from Latin America. 
In Carnegie-Rochester conference series on public policy, Vol. 45, pp. 129-162, North-Holland.

Ng, S., \& Perron, P. (1995) Unit root tests in ARMA models with datadependent methods for the selection of the truncation lag. Journal of the American Statistical Association, 90(429), 268-281.

Norris, E. D., \& Feldstein, A. (2003) An analysis of the Underground Economy and Its Macroeconomic Consequences, International Monetary Fund (IMF), Working Paper, No. 23.

Phillips, P. C. B., \& Perron, P. (1988) Testing for a Unit Root in Time Series Regression, Biometrika, 75(2), 335-346.

Porta, Rafael La and Shleifer, Andrei (2014) Informality and Development, Journal of Economic Perspectives, Vol. 28, No. 3, Summer, P.109-126.

Said, Said E. \& Dickey, D. (1984) Testing for Unit Roots in Autoregressive-Moving Average Models of Unknown Order, Biometrika, 71(3), 599-607.

Savaşan, Fatih (2003) Modeling the Underground Economy in Turkey: Randomized Response and MIMIC Models. The Journal of Economics, 29(1), 49-76.

Schneider, F. (2005) Shadow economies around the world: what do we really know? European Journal of Political Economy, 21(3), 598642.

Schneider, F., \& Enste, D. H. (2000) Shadow economies: size, causes, and consequences. Journal of economic literature, 38(1), 77-114.

Schneider, Friedrich and Enste, Dominik (2002) Hiding in the Shadows: The Growth of the Underground Economy, IMF Economic Issues, No. 30, March.

Schwert, G. W. (2002) Tests for unit roots: A Monte Carlo investigation. Journal of Business \& Economic Statistics, 20(1), 517.

Spiro, Peter S. (1997) Taxes, Deficit, and the Underground Economy, In Lippert, Owen \& Walker, Michael, The Underground Economy: global Evidence of its Size and Impact. The Fraser Institute, Vancouver, British Columbia, Canada. 